\title{
Inference of accumulation-rate patterns from deep layers in glaciers and ice sheets
}

\author{
Edwin D. WADDINGTON, ${ }^{1}$ Thomas A. NEUMANN, ${ }^{1,2}$ Michelle R. KOUTNIK, ${ }^{1}$ \\ Hans-Peter MARSHALL, ${ }^{*}$ David L. MORSE ${ }^{1}$ \\ ${ }^{1}$ Department of Earth and Space Sciences, University of Washington, Seattle, Washington 98195-1310, USA \\ E-mail: edw@ess.washington.edu \\ ${ }^{2}$ Department of Geology, University of Vermont, Burlington, Vermont 05405-0122, USA
}

\begin{abstract}
The spatial pattern of accumulation rate can be inferred from internal layers in glaciers and ice sheets. Non-dimensional analysis determines where finite strain can be neglected ('shallow-layer approximation') or approximated with a local one-dimensional flow model ('local-layer approximation'), and where gradients in strain rate along particle paths must be included ('deep layers'). We develop a general geophysical inverse procedure to infer the spatial pattern of accumulation rate along a steady-state flowband, using measured topography of the ice-sheet surface, bed and a 'deep layer'. A variety of thermomechanical ice-flow models can be used in the forward problem to calculate surface topography and ice velocity, which are used to calculate particle paths and internal-layer shapes. An objective tolerance criterion prevents over-fitting the data. After making site-specific simplifications in the thermomechanical flow algorithm, we find the accumulation rate along a flowband through Taylor Mouth, a flank site on Taylor Dome, Antarctica, using a layer at approximately $100 \mathrm{~m}$ depth, or $20 \%$ of the ice thickness. Accumulation rate correlates with ice-surface curvature. At this site, gradients along flow paths critically impact inference of both the accumulation pattern, and the depth-age relation in a $100 \mathrm{~m}$ core.
\end{abstract}

\section{INTRODUCTION}

\subsection{Radar layers}

Ice-penetrating radars (IPR) were developed to measure ice thickness in polar ice sheets (Paren and Robin, 1975). These radars can also detect internal layers, which are commonly interpreted as horizons of constant age (isochrones). These layers can be used to infer the history of ice sheets, including migration of ice divides (Nereson and others, 1998), changes in thickness, and initiation of new ice divides (e.g. Conway and others, 1999; Nereson and Raymond, 2001). The depths of these layers also provide information on accumulationrate patterns; however, the most appropriate technique to extract this information depends on the amount of strain that the ice has experienced.

\subsection{Strain regimes}

In the uppermost few percent of an ice sheet, groundpenetrating radars (GPR) also detect layers, and local accumulation rate can be viewed as directly proportional to ice-equivalent layer depth (e.g. Pinglot and others, 2001; Spikes and others, 2004). We call this the shallow-layer approximation (SLA).

Since older layers reflect longer-term patterns of climate, there is also strong motivation to extract accumulation patterns from these older layers. As the depth of an internal layer increases to a larger fraction of the total ice thickness, the ice above that layer has been subjected to increasing total strain as a result of ice flow. The SLA is no longer appropriate for layers buried by more than a few percent of the ice thickness. For these layers, a layer-thinning correction must

\footnotetext{
* Present address: Institute of Arctic and Alpine Research, University of Colorado, Boulder, Colorado 80309-0450, USA.
}

be applied. Accumulation rates have been extracted from these layers by integrating local vertical strain rates in a onedimensional (1-D) flow model to get a strain correction (e.g. Morse and others, 1999; Vaughan and others, 1999; Fahnestock and others, 2001; Kanagaratnam and others, 2001; Pälli and others, 2002; Leysinger Vieli and others, 2004; Siegert and Payne, 2004). We call this the local-layer approximation (LLA). Using the LLA is equivalent to assuming that there are no horizontal gradients in geometry and strain rate, or that a sandwich model as described by Reeh (1989) adequately represents the flow.

However, even the LLA becomes inadequate for deeper layers, where horizontal gradients in accumulation rate and strain rate along particle paths have significantly affected layer depths. Extraction of accumulation-rate information from these layers is a challenging problem that requires a full treatment of ice flow and accumulated strain along particle paths. We define these as deep layers for the purpose of inferring accumulation-rate patterns.

In section 6, we derive non-dimensional numbers that determine whether the SLA, the LLA or a deep-layer treatment should be used for any particular layer. In this paper, we describe a general inverse method to extract accumulation-rate information from deep layers.

\subsection{Previous work}

Several previous studies have avoided the limitations of the LLA by using flowline particle-tracking models to calculate layer shapes. Nereson and others (2000) used a twodimensional (2-D) flow model to estimate the spatial pattern of accumulation across Siple Dome, Antarctica. They approximated the spatial variation of accumulation rate with an arctangent function, and used an inverse procedure to select the two parameters defining its amplitude and length 
scale on each side of the divide. Baldwin and others (2003) tracked particles backward along trajectories in a steady-state model, starting from two internal layers of known ages in the Greenland ice sheet. They used mismatches between their modelled surface and the observed surface to infer corrections to the measured accumulation pattern. The adjustment algorithm allowed only corrections that varied slowly over length scales greater than the particle-path lengths. Variability over shorter distances could be incorporated only through the initial guess, which was based on previously measured accumulation rates. The algorithm could not create, correct or remove short-wavelength structures. In practice, application of the method was limited to relatively young layers with short particle paths, or to regions with low spatial gradients in accumulation rate, where layer ages are already known. Parrenin and others (2006) presented analytical solutions for steady-state shapes of deep layers. Hindmarsh and others (2002) used a formal optimal-filter inverse approach to match observed layers, and Martín and others (2006) used a controlmethod approach to infer a spatial pattern of accumulation rate from internal layers. Their work bears a strong conceptual connection to the work that we address here, although it used a different inverse approach.

Here, we describe a general, robust procedure using geophysical inverse theory (e.g. Menke, 1989; Parker, 1994; Aster and others, 2005) to recover the spatial pattern and magnitude of accumulation rate along a flowband, using a deep layer. The method can recover the information on spatial patterns of accumulation rate as retained in internallayer geometry, without additional assumptions about scales of variability, or layer age.

\subsection{Forward and inverse problems}

The forward problem is the calculation of ice-velocity and ice-temperature fields, ice-surface topography and shapes of internal layers. The forward problem is described by a set of conservation and constitutive equations, boundary conditions and parameter values. Those boundary conditions include the spatial pattern of accumulation rate. In the corresponding inverse problem, we know the equations, and we may know some or all of the boundary conditions, and some or all of the parameter values. We incorporate data into the solution procedure in order to infer the remaining unknown boundary conditions and parameters.

In our specific inverse problem, we can use surface-profile and layer-depth information, as well as other data (if available), such as point measurements of surface velocity and accumulation rate, in order to find a smooth accumulationrate profile and other model parameters that together produce an expected level of mismatch between the data and corresponding values calculated using these model parameters in the forward problem.

Since fully general inverse procedures can be computationally expensive, it is desirable to customize and reduce the complexity of the inverse procedure by exploiting any simplifications that can be justified in each particular application. After describing the inverse procedure in its general form, we apply the method to an internal layer observed along a flowline near Taylor Dome, Victoria Land, Antarctica. By introducing additional approximations that are appropriate to this particular flowline, we illustrate how the general procedure can be simplified for this particular field site.

\section{Notation}

We use boldface lower-case type to represent vectors, and single subscripts to indicate their components. We use boldface upper-case type to represent matrices, and double subscripts to denote their components.

\section{FORWARD PROBLEM}

We solve our forward problem to find ice-surface elevation, ice velocities and layer shapes, using a flowband with steadystate surface, velocities and temperature. The flowband has variable width $W(x)$, where $x$ follows the flow direction, $y$ is horizontal and transverse to the flow, and $z$ is the vertical coordinate. $S(x)$ is the ice-equivalent surface elevation, $B(x)$ is the bed elevation, $(S-z)$ is the depth below the surface, and $H(x)=S(x)-B(x)$ is ice-equivalent thickness. Our model can be applied to any limited region of an ice sheet, including those that do not include an ice divide. Our formulation is designed to allow a wide range of thermomechanical calculations to be adapted to it.

\subsection{Flowband geometry}

With the assumption that flow direction is controlled by the local surface slope, flowlines can be defined as trajectories that are everywhere parallel to the gradient of the measured surface topography. When surface-velocity measurements are also available, that assumption can be relaxed, and flowlines can be defined as trajectories that are everywhere parallel to the measured velocities. A flowband is the volume bounded by two vertical surfaces directly beneath two nearby flowlines. We use a steady-state flowband of variable width $W(x)$, where $x$ follows the ice-flow direction along the center of the flowband, $y$ is horizontal and transverse to the flow, and $z$ is elevation. The bed elevation $B(x)$ must also be known along the flowband. Bed elevation $B(x)$, ice thickness $S(x)$, and the components of velocity $\mathbf{v}=(u, v, w)$ in the $x$ and $z$ directions are assumed to be uniform in the $y$ direction transverse to the central flowline. In solving our forward problem, we calculate the steady-state ice-equivalent ice-surface topography $S(x)$, and the steady-state flow field v everywhere in the flowband. The velocity is tangential to the vertical boundaries of the flowband, and $u$ and $w$ are invariant in the $y$ direction across the flowband. Depth below the surface is $(S(x)-z)$, and ice-equivalent thickness is $H(x)=S(x)-B(x)$.

\subsection{Rheological parameters}

When the coordinates $(x, y, z)$ are written in index notation as $\left(x_{1}, x_{2}, x_{3}\right)$, ice strain rate $\dot{\epsilon}_{i j}=1 / 2\left(\partial u_{i} / \partial x_{j}+\partial u_{j} / \partial x_{i}\right)$ is calculated from a temperature-dependent flow law (e.g. Glen, 1958; Paterson, 1994, ch. 5), using

$$
\dot{\epsilon}_{i j}=E A(\theta) \tau_{\mathrm{e}}^{n-1} \tau_{i j}
$$

where $i$ and $j$ can take values from 1 to $3, \tau_{i j}$ is the deviatoric stress tensor, $\tau_{\mathrm{e}}$ is its second invariant, $n=3$ is the flowlaw exponent, and $\theta$ is temperature. $A(\theta)$ is a temperaturedependent softness or fluidity parameter; $A(\theta)$ is generally written as $A_{o} \exp (-Q / R \theta)$ (Paterson, 1994, p. 86), where $Q=60 \mathrm{~kJ} \mathrm{~mol}^{-1}$ is the activation energy for creep, and $R=8.314 \mathrm{~J} \mathrm{~mol}^{-1} \mathrm{~K}^{-1}$ is the gas constant. We use $A_{\mathrm{o}}=$ $4.0 \times 10^{-4} \mathrm{kPa}^{-3} \mathrm{~s}^{-1}$, the value derived from Paterson (1994, p. 97 , table 5.2) for temperatures below $-10^{\circ} \mathrm{C}$. At a given temperature $\theta$, the rheological behavior of ice in our model is 
fully determined by a single adjustable model parameter, the enhancement factor $E$. Under other circumstances, $E$ may vary spatially (e.g. to represent a contrast between ice-age and interglacial ice). If ice is treated as an anisotropic material, a different constitutive relation may replace Equation (1), and additional parameters may be introduced.

\subsection{Thermomechanical flow calculations}

Calculation of surface topography and velocity requires calculation of the temperature field $\theta$, because strain rate $\dot{\epsilon}_{i j}$ in ice (Equation (1)), basal-sliding rate, and basal-melting rate all depend on the temperature distribution. In many cases, the temperature calculations also depend on ice flow. Any steady-state thermomechanical calculation also requires a specified ice thickness at one location to provide a boundary condition. For example, we might use the measured ice thickness at an ice-core site. When the parameters controlling mass balance, rheological properties and thermal conditions have been specified, the forward problem can be solved using any of a wide variety of ice-flow models to calculate the steady-state surface topography, ice velocity, and temperature. These thermomechanical calculations generally proceed by integrating a rheological relation such as Equation (1), while simultaneously solving a heat-flow equation for the temperature. Because the required complexity of these calculations can be site-specific, we do not describe a general thermomechanical calculation procedure. In Appendix B, we describe a specific simplified thermomechanical calculation procedure that is appropriate for our site at Taylor Mouth, Antarctica.

\subsection{Kinematic representation of velocity}

For a given accumulation-rate pattern $\dot{b}(x)$ and basal meltingrate pattern $\dot{m}(x)$, both expressed in ice-equivalent units, the steady-state ice-flux profile $q(x)$ along the flowband is given by

$$
q(x)=q_{\text {in }}+\int_{x_{\text {in }}}^{x}(\dot{b}(\zeta)-\dot{m}(\zeta)) W(\zeta) \mathrm{d} \zeta,
$$

where $q_{\text {in }}$ is the flux entering or leaving the flowband at $x=x_{\text {in }}$. At any location $x$, the flowband has cross-sectional area $W(x) \times H(x)$. The ice flux $q(x)$ through the cross-section is also proportional to $\bar{u}(x)$, the horizontal velocity $u(x, z)$ averaged over that cross-section, through

$$
q(x)=W(x) \int_{B(x)}^{S(x)} u(x, z) d z=W(x) H(x) \bar{u}(x) .
$$

The distribution of velocity with depth depends on the basal boundary conditions and on the selected thermomechanical ice-flow algorithm. In order to make the inverse procedure independent of details of specific algorithms for thermomechanical flow calculations, and to standardize procedures for particle tracking, we represent horizontal velocities $u(x, z)$ along a vertical profile at any position $x$ as the product of the depth-averaged velocity $\bar{u}(x)$, and a non-dimensional shape function $\phi(x, \hat{z})$ (Reeh, 1988), which describes how the horizontal velocity varies with depth, giving

$$
u(x, z)=\bar{u}(x) \phi(x, \hat{z}),
$$

where $\hat{z}$ is normalized non-dimensional height above the bed, given by

$$
\hat{z}=\frac{z-B(x)}{S(x)-B(x)} .
$$

The horizontal-velocity shape function $\phi(x, \hat{z})$ is produced by the forward algorithm, in which many different thermomechanical ice-flow models can be used. The sophistication required of the thermomechanical model depends on the characteristics of the particular site under investigation. One approach is to use the shape functions $\phi(x, \hat{z})$ generated by a simple kinematic approximation such as the Dansgaard and Johnsen (1969) model. A second approach is to use shape functions based on the shallow-ice approximation (SIA; e.g. Paterson, 1994, p. 262), incorporating depth-varying temperature. A third approach, followed by Nereson and others (1998) and by Nereson and Waddington (2002), is to use polynomial approximations to the velocity profiles produced by a finite-element momentum-conservation flow model (e.g. Raymond, 1983; Hvidberg, 1996) that incorporates all stress components for plane strain, and incorporates the influence of temperature on strain rates.

The strain rate transverse to the flowband is (e.g. Waddington, 1982)

$$
\frac{\partial v(x, z)}{\partial y}=\frac{1}{W(x)} \frac{\mathrm{d} W}{\mathrm{~d} x} u(x, z)
$$

The vertical velocity $w(x, z)$ can then be recovered from incompressibility,

$$
\frac{\partial w}{\partial z}=-\left(\frac{\partial u}{\partial x}+\frac{\partial v}{\partial y}\right)
$$

At the bed $(z=B(x)$, or $\hat{z}=0)$, the vertical velocity is

$$
w(x, B(x))=-\dot{m}(x)+\bar{u}(x) \phi(x, 0) \frac{\mathrm{d} B}{\mathrm{~d} x},
$$

where the first term represents vertical motion due to basal melting, and the second term represents vertical motion due to sliding over basal topography. Melting and sliding can be calculated by appropriate equations or algorithms in the forward problem. A vertical integration of Equation (7) from the bed, together with Equations (2-6) leads to an expression for the vertical velocity:

$$
\begin{aligned}
w(x, z)= & -(\dot{b}(x)-\dot{m}(x)) \psi(x, \hat{z})-\dot{m}(x) \\
& +u(x, z)\left((1-\hat{z}) \frac{\mathrm{d} B}{\mathrm{~d} x}+\hat{z} \frac{\mathrm{d} S}{\mathrm{~d} x}\right) \\
& -\bar{u}(x) H(x) \int_{0}^{\hat{z}} \frac{\partial \phi(x, \hat{\zeta})}{\partial x} \mathrm{~d} \hat{\zeta},
\end{aligned}
$$

where

$$
\psi(x, \hat{z})=\int_{0}^{\hat{z}} \phi(x, \hat{\zeta}) \mathrm{d} \hat{\zeta}
$$

In the absence of basal melting, basal sliding and horizontal gradients in surface elevation, bed elevation and horizontalvelocity shape function, the first term fully describes the vertical velocity; hence, $\psi(x, \hat{z})$ is sometimes called a verticalvelocity shape function.

\subsection{Particle paths and layers}

Finally, particle paths are found by integrating the velocity field in Equations (4) and (9), viewed as the material derivative of particle position. To find the depth $h^{(\mathrm{m})}(x, \mathcal{A})\left({ }^{\prime} \mathrm{m}\right.$ ' for modelled) of a modelled layer of age $\mathcal{A}$, we join the end points of paths at time $t=0$ for a number of particles that started at the ice-sheet surface $S(x)$, at points $x=x_{j}^{(\mathrm{s})},\left({ }^{\prime} \mathrm{s}\right.$ ' for surface) at $t=-\mathcal{A}$. 


\section{INVERSE PROBLEM}

\subsection{Strategy}

The standard approach when solving a geophysical inverse problem (see, e.g., Menke, 1989; Parker, 1994) is to find a 'model'. The model is a vector $\mathbf{p}$ comprising a set of $N_{p}$ model parameters $\left(\left\{p_{j}, j=1, \ldots, N_{p}\right\}\right)$, which are required in the solution of the forward problem. Many forward problems are diffusive in character, so that details of the initial conditions are forgotten over time, and details in boundary conditions are attenuated with distance from the boundaries. When those initial or boundary conditions are model parameters that we wish to recover by solving an inverse problem, the data may contain enough information to recover only weighted averages of those initial or boundary values. The data may also retain insufficient information to reject large and physically unrealistic oscillations in those initial or boundary values, and spurious structures can appear in the solution. Therefore, we must stabilize, or regularize, the solution to find a preferred model that is also physically plausible (e.g. Parker, 1994; Truffer, 2004; Aster and others, 2005). One way to regularize an inverse problem is to require that the solution be smoothly varying.

The inversion procedure selects a model $\mathbf{p}$ that has a minimum magnitude, as measured by some scalar functional called a 'model norm', which we write as $\|\mathbf{p}\|$. This model norm provides the means by which we impose regularization on the problem. For example, if the model is the spatial accumulation-rate distribution, then we might choose to minimize a model norm where $\|\mathbf{p}\|$ is a roughness measure. Minimizing a norm that measures roughness allows us to select a spatially smooth accumulation-rate solution from among a large number of possible solutions, most of which may be less physically plausible. Other choices of model norm are also possible.

Because the data contain measurement errors, and because our formulation of the forward problem may be imperfect, we should not fit the data too closely. Over-fitting data can also produce a model that contains more structure (e.g. spatial oscillations in the accumulation-rate profile) than is necessary to 'explain' the data, within their uncertainties, and this structure is likely to be spurious. We prefer to seek robust models, in which all structure is required by the data, at the expense of potentially failing to detect model structure that might be present but which is not absolutely required by the data. The preferred model should reproduce the $N_{\mathrm{d}}$ observations $\left(\left\{o_{i}^{(\mathrm{d})}, i=1, \ldots, N_{\mathrm{d}}\right\}\right)$ (' $\mathrm{d}$ ' for data) at an acceptable tolerance level $T$, which is determined by the statistical uncertainties in the data. To quantify data misfit, we also define a scalar 'data norm' $\|\mathbf{d}\|$, which is a measure of non-dimensional mismatch between the data vector $\mathbf{o}^{(\mathrm{d})}$ and the model predictions $\mathbf{o}^{(\mathrm{m})}$ of the corresponding observables from a solution of the forward problem.

This mismatch criterion

$$
\|\mathbf{d}\|^{2}-T^{2}=0
$$

forms a constraint that must be satisfied by the model $\mathbf{p}$, as its model norm $\|\mathbf{p}\|$ is minimized. There is extensive literature on constrained minimization of quadratic functions (e.g. Morse and Feshbach, 1953). We define a performance index $I_{p}$ :

$$
I_{p}=\|\mathbf{p}\|^{2}+\nu\left(\|\mathbf{d}\|^{2}-T^{2}\right)
$$

where $\nu$ is a positive Lagrange multiplier. In the language of the calculus of variations, the vector $\mathbf{p}$ and value of $\nu$ that minimize $\|\mathbf{p}\|^{2}$ subject to the constraint in Equation (11) are found at a stationary point of $I_{p}$ in $(\mathbf{p}, \nu)$ space. This stationary point can be found by solving the set of $N_{p}+1$ equations

$$
\begin{aligned}
& \frac{\partial I_{p}}{\partial p_{j}}=0 \quad j=1, \ldots, N_{p} \\
& \frac{\partial I_{p}}{\partial \nu}=0 .
\end{aligned}
$$

The $N_{p}$ equations in (13) lead naturally to the normal equations for a least-squares minimization problem. In the language of inverse theory, the Lagrange multiplier $\nu$ is called a trade-off parameter because it expresses the 'best' balance, or trade-off, between minimizing the model norm $\|\mathbf{p}\|$, and fitting the data through the data norm $\|\mathbf{d}\|$, as expressed in Equation (11). The model solution vector $\mathbf{p}$ is the smoothest result that also fits the data at tolerance level $T$ (not better and not worse).

\subsection{Model parameters}

We know the bed elevation $B(x)$, and the width $W(x)$ along the flowband. We also know the mean annual temperature at the ice-sheet surface, and the ice thickness along the flowband. In general, the model parameters $\mathbf{p}$ that we wish to find then comprise the accumulation-rate profile $\dot{b}(x)$, the ice flux $q_{\text {in }}$ entering or leaving the flowband at one boundary, the age $\mathcal{A}$ of the layer, the ice thickness $H_{0}$ at one point along the flowband, the flow enhancement factor $E$ and the geothermal flux $q_{\text {geo. }}$. If the bed reaches the pressure-melting temperature, then basal sliding $u(x, 0)$ and basal melting $\dot{m}(x)$ must be included in the parameter list, either directly as spatial profiles, or through a few parameters that allow their values to be calculated in the forward algorithm. In some situations, the inverse procedure can be simplified by assigning fixed values to some of these quantities, rather than retaining them as adjustable model parameters.

So that we can reduce $\mathbf{p}$ to a finite number of parameters, we represent the accumulation pattern $\dot{b}(x)$ by a piecewiselinear function. We select $N_{\mathrm{n}}$ nodes $\left\{x_{j}^{(\mathrm{n})}, j=1, \ldots, N_{\mathrm{n}}\right\}$ (' $\mathrm{n}$ ' for nodes). Then, the accumulation-rate parameters are the values $\dot{b}_{j} \equiv \dot{b}\left(x_{j}^{(\mathrm{n})}\right)$ of accumulation rate at nodes $x_{j}^{(\mathrm{n})}$.

\subsection{Model norm}

Since we expect that the accumulation rate varies smoothly along the flowband, we choose to minimize a model norm that measures model roughness. We represent global model roughness in scalar form by the square of the second derivative $\mathrm{d}^{2} \dot{b} / \mathrm{d} x^{2}$ of the accumulation rate $\dot{b}(x)$, integrated along the flowband. Because $\dot{b}(x)$ is piecewise-linear, its second derivative is actually zero everywhere except at the nodes $x_{j}^{(\mathrm{n})}$, where it can be infinite. So, instead of using the actual curvature of the piecewise-linear function, we estimate smoothness in the vicinity of node $x_{j}^{(\mathrm{n})}$ by attributing a uniform curvature to the $x$ interval of length $\Delta x_{j}=\left(x_{j+1}^{(\mathrm{n})}-\right.$ $\left.x_{j-1}^{(\mathrm{n})}\right) / 2$, which joins the midpoints of two adjacent linear segments, i.e. in the vicinity of node $x_{j}^{(\mathrm{n})}$, the upstream and downstream boundaries for intervals of uniform curvature are $\left(x_{j-1}^{(\mathrm{n})}+x_{j}^{(\mathrm{n})}\right) / 2$ and $\left(x_{j}^{(\mathrm{n})}+x_{j+1}^{(\mathrm{n})}\right) / 2$. We use a standard finite-difference representation of a uniform second derivative in this interval, based on the accumulation-rate model 
parameters $\dot{b}_{j}$, and non-dimensionalized with a characteristic length scale $L^{(\mathrm{c})}$ (e.g. characteristic ice thickness, or characteristic length scale of surface undulations, both of which can be related to accumulation-rate variations), and a characteristic accumulation rate $\dot{b}^{(\mathrm{c})}$ (e.g. the value from a core site), giving non-dimensional curvature in the $j$ th such interval as

$$
c_{j}=\frac{L^{(\mathrm{c})^{2}}}{\dot{b}^{(\mathrm{c})}} \frac{\mathrm{d}^{2} \dot{b}}{\mathrm{~d} x^{2}}=\frac{L^{(\mathrm{c})^{2}}}{\dot{b}^{(\mathrm{c})}}\left[\frac{\left(\frac{\dot{b}_{j+1}-\dot{b}_{j}}{x_{j+1}^{(\mathrm{n})}-x_{j}^{(\mathrm{n})}}\right)-\left(\frac{\dot{b}_{j}-\dot{b}_{j-1}}{x_{j}^{(\mathrm{n})}-x_{j-1}^{(\mathrm{n})}}\right)}{\Delta x_{j}}\right] .
$$

If the profiles of basal sliding $u(x, 0)$ or melting $\dot{m}(x)$ are included as model parameters, they can be regularized in a similar way through a smoothness condition analogous to Equation (15); however, if basal sliding or melting are calculated in the forward algorithm from a few physical parameters, rather than specified directly as series of unknown model parameters, a different treatment is needed. None of the parameters $q_{\text {in }}, \mathcal{A}, H_{0}, E$ or $q_{\mathrm{geo}}$ or parameters for calculating basal sliding or melting profiles can be included in a smoothness measure, because they do not naturally belong in a spatial sequence with other parameters. However, we can include each of these parameters in the model norm by penalizing its distance from an expected value $p_{j}^{(\mathrm{e})}$ (' $\mathrm{e}^{\prime}$ for expected). An initial guess for the ice flux $q_{\text {in }}^{(0)}$ can be derived from rough ideas about the upstream catchment area and accumulation rate. An initial guess for the layer age $\mathcal{A}^{(0)}$ might be derived from a nearby ice core. An initial guess for $H_{0}^{(0)}$ could be its measured value, and an initial estimate for the enhancement factor could be $E^{(0)}=1$. An initial guess for the geothermal flux $q_{\text {geo }}^{(0)}$ could be derived from knowledge of the tectonic setting. In the model norm, distance from this expected value is expressed in non-dimensional units measured by a characteristic acceptable deviation $\delta p_{j}^{(\mathrm{c})}$, i.e.

$$
c_{j}=\frac{p_{j}-p_{j}^{(\mathrm{e})}}{\delta p_{j}^{(\mathrm{c})}} .
$$

Assigning a large value to $\delta p_{j}^{(\mathrm{c})}$ expresses limited conviction in our expectations, and allows the model to deviate more readily from the corresponding expectation $p_{j}^{(\mathrm{e})}$, for a given contribution of model parameter $p_{j}$ (through $c_{j}$ ) to the model norm

$$
\|\mathbf{p}\|^{2}=\sum_{j=1}^{N_{\mathrm{p}}}\left(w_{j}^{(\mathrm{c})}\right)^{2} c_{j}^{2} .
$$

In Equation (17), the positive non-dimensional weights $\left(w_{j}^{(\mathrm{c})}\right)^{2}$ depend on the type of contribution $c_{j}$. When $c_{j}$ represents the uniform curvature of accumulation rate in the vicinity of node $x_{j}^{(\mathrm{n})}$ (Equation (15)), its squared value $c_{j}^{2}$ is integrated over the non-dimensional $x$ interval $\Delta x_{j} / \overline{\Delta x}$, where $\overline{\Delta x}=\left(x_{N}^{(\mathrm{n})}-x_{1}^{(\mathrm{n})}\right) /\left(N_{\mathrm{n}}-1\right)$ is the average length of these intervals $\Delta x_{j}$ along the flowband, leading to the weight $w_{j}^{(\mathrm{c})}=\left(\Delta x_{j} / \overline{\Delta x}\right)^{1 / 2}$. When $j$ identifies one of the other model parameters (e.g. $q_{\text {in }}, \mathcal{A}, H_{0}, E$ or $q_{\text {geo }}$ ), then $w_{j}^{(\mathrm{c})}=1$.

The contributions $c_{j}$ to the model norm can be viewed as model residuals, in the sense that they measure the degree to which the parameters fail to match expectations, either of zero curvature in Equation (15), or of a preconceived value $p_{j}^{(\mathrm{e})}$ in Equation (16).

\subsection{Data norm}

The inversion procedure must incorporate data that constrain the current geometry, and data that constrain rates of flow in the forward problem. The geometric observations comprise the depth $h^{(\mathrm{d})}(x)$ ('d' for data) of a layer measured by ice-penetrating radar, and surface elevation $S^{(\mathrm{d})}(x)$ measured by global positioning system (GPS) or optical survey techniques. During the inversion procedure, these profiles are resampled to produce datasets $h_{i}^{(\mathrm{d})} \equiv h^{(\mathrm{d})}\left(x_{i}^{h}\right)$ at $N_{h}$ positions $\left\{x_{i}^{h}, i=1, \ldots, N_{h}\right\}$, and surface elevation $S_{i}^{(\mathrm{d})} \equiv S^{(\mathrm{d})}\left(x_{i}^{S}\right)$ at $N_{S}$ positions $\left\{x_{i}^{S}, i=1, \ldots, N_{S}\right\}$.

The rate-constraining data comprise observations of accumulation rate $\dot{b}_{i}^{(\mathrm{d})} \equiv \dot{b}^{(\mathrm{d})}\left(x_{i}^{\dot{b}}\right)$ at $N_{\dot{b}}$ positions $\left\{x_{i}^{\dot{b}}, i=\right.$ $\left.1, \ldots, N_{b}\right\}$, and ice velocity $u_{i}^{(\mathrm{d})} \equiv u^{(\mathrm{d})}\left(x_{i}^{u}\right)$ at $N_{u}$ positions $\left\{x_{i}^{u}, i=1, \ldots, N_{u}\right\}$ along the flowband (if available). The observations of each type can be written as a vector, $\mathbf{h}^{(\mathrm{d})}, \mathbf{S}^{(\mathrm{d})}$, $\dot{\mathbf{b}}^{\text {(d) }}$ and $\mathbf{u}^{(\mathrm{d})}$, and these vectors are then combined to form the vector of observations $\mathbf{o}^{(\mathrm{d})}$ of length $N_{d}=N_{h}+N_{S}+N_{b}+N_{u}$.

For each observation $o_{i}^{(\mathrm{d})}$, there is also a corresponding quantity calculated in the forward problem; we call this the modelled observable $o_{i}^{(\mathrm{m})}$, and we form the non-dimensional residual

$$
r_{i}=\frac{o_{i}^{(\mathrm{m})}-o_{i}^{(\mathrm{d})}}{\sigma_{i}^{(\mathrm{d})}}
$$

comprising the mismatch, divided by the standard error $\sigma_{i}^{(\mathrm{d})}$ of the measurement.

We can apply Equation (18) to find residuals for each data type; these residuals can be written as column vectors $\mathbf{r}^{h}, \mathbf{r}^{S}$, $\mathbf{r}^{\dot{b}}$ and $\mathbf{r}^{u}$, whose elements are $r_{i}^{h} \equiv r^{h}\left(x_{i}^{h}\right), r_{i}^{S} \equiv r^{S}\left(x_{i}^{S}\right), r_{i}^{\dot{b}} \equiv$ $r^{\dot{b}}\left(x_{i}^{\dot{b}}\right)$, and $r_{i}^{u} \equiv r^{u}\left(x_{i}^{u}\right)$, respectively. When these residuals are all combined into a single residual vector $\mathbf{r}$, the global mismatch is expressed by the data norm $\|\mathbf{d}\|$ defined by

$$
\|\mathbf{d}\|^{2}=\sum_{i=1}^{N_{\mathrm{d}}} r_{i}^{2}=\sum_{i=1}^{N_{\mathrm{d}}}\left[\frac{\left(o_{i}^{(\mathrm{m})}-o_{i}^{(\mathrm{d})}\right)}{\sigma_{i}^{(\mathrm{d})}}\right]^{2} .
$$

If the non-dimensional mismatches in Equation (18) were linearly independent and normally distributed, then the tolerance $T$ to which we should match the data (using Equation (11)) could be represented through the expected value of the square root of a sum of squares of $N$ realizations of a process with zero mean and unit variance (Parker, 1994, p. 124), i.e.

$$
T \approx N_{\mathrm{d}}^{1 / 2}\left[1-\frac{1}{4 N_{\mathrm{d}}}+\frac{1}{32 N_{\mathrm{d}}^{2}}+O\left(N_{\mathrm{d}}^{-3}\right)\right] .
$$

Although the residuals may not be strictly independent and normally distributed, Equation (20) provides a useful estimate for $T$, and we can test its validity after we have found a solution vector p. (See section 5.1.)

\subsection{Solution procedure}

Equation (13) can be written in terms of model residuals $c_{j}$ from Equation (17), which depend on the model parameters $p_{j}$ through Equations (15) and (16), and data residuals $r_{j}$ from Equation (19), which depend on the model parameters $p_{j}$ 
through the model predictions $O_{i}^{(\mathrm{m})}$ of observable quantities in Equation (18). The $o_{i}^{(\mathrm{m})}$ predictions are not linear functions of the model parameters $p_{j}$, so Equations (13) and (14) cannot be solved directly. In order to find the 'best' model-parameter vector $\mathbf{p}$ and trade-off parameter $\nu$, we start with a trial solution $\mathbf{p}^{(0)}$ and $\nu^{(0)}$. Initial guesses for parameters $q_{\text {in }}, \mathcal{A}, H_{0}, E$ and $q_{\text {geo }}$ can be the expected values $p_{j}^{(\mathrm{e})}$ for those parameters. For example, the flux $q_{\text {in }}^{(0)}$ can be estimated from rough ideas about the upstream catchment area and accumulation rate. An initial estimate for the enhancement factor is $E^{(0)}=1$. We can use the SLA (sections 1.2 and 6.5) to estimate the accumulation-rate parameters. Dividing the layer-depth data $h^{(\mathrm{d})}\left(x_{j}^{(\mathrm{n})}\right)$ at the nodes $x_{j}^{(\mathrm{n})}$, by the initial estimate $\mathcal{A}^{(0)}$ of the layer age, gives $\dot{b}_{j}^{(0)}=h^{(\mathrm{d})}\left(x_{j}^{(\mathrm{n})}\right) / \mathcal{A}^{(0)}$. An initial guess for the trade-off parameter could be $\nu^{(0)}=1.0$. However, there are two problems with this initial guess.

First, when used with $\nu^{(0)}$ in the forward model, the modelparameter vector $\mathbf{p}^{(0)}$ may not produce a minimum $I_{p}$ in Equation (12). Therefore, the estimate of the model-parameter vector $\mathbf{p}$ must be improved by iteration. At iteration $k$, the unknown parameters $\mathbf{p}^{(k)}$ that would satisfy Equations (13) are expressed in terms of the known current estimates $\mathbf{p}^{(k-1)}$, and unknown corrections $\Delta \mathbf{p}^{(k)}$ :

$$
\mathbf{p}^{(k)}=\mathbf{p}^{(k-1)}+\Delta \mathbf{p}^{(k)} .
$$

Here, we outline the solution procedure. Details are given in Appendix A. Expanding the unknown updated model residuals $\mathbf{c}^{(k)}$ and data residuals $\mathbf{r}^{(k)}$ in Equations (17) and (19) in terms of the unknown parameter corrections $\Delta \mathbf{p}^{(k)}$ in the vicinity of $\mathbf{c}^{(k-1)}$ and $\mathbf{r}^{(k-1)}$ respectively, produces a set of $\left(N_{p}+N_{d}\right)$ linear equations for the parameter corrections $\Delta \mathbf{p}^{(k)}$. In block-matrix form, the equations to be solved are

$$
\left[\begin{array}{cc}
\mathbf{W}_{\mathrm{c}} & \mathbf{0} \\
\mathbf{0} & \mathbf{W}_{\mathrm{r}}
\end{array}\right]\left[\begin{array}{l}
\mathbf{J}_{\mathrm{c}} \\
\mathbf{J}_{\mathrm{r}}
\end{array}\right][\Delta \mathbf{p}]=-\left[\begin{array}{cc}
\mathbf{W}_{\mathrm{c}} & \mathbf{0} \\
\mathbf{0} & \mathbf{W}_{\mathrm{r}}
\end{array}\right]\left[\begin{array}{l}
\mathbf{c} \\
\mathbf{r}
\end{array}\right],
$$

where $\mathbf{c}$ and $\mathbf{r}$ are the parameter-residual and data-residual vectors, and $\mathbf{J}_{\mathrm{C}}$ and $\mathbf{J}_{\mathrm{r}}$ are Jacobian matrices whose elements in row $i$ and column $j$ are the partial derivatives $\partial c_{i} / \partial p_{j}$ and $\partial r_{i} / \partial p_{j}$, respectively. In general, these partial derivatives must be evaluated numerically by perturbing each model parameter $p_{j}$ in turn, and recalculating all the model residuals $c_{i}$ and data residuals $r_{i}$. Diagonal matrices $\mathbf{W}_{\mathrm{c}}$ and $\mathbf{W}_{\mathrm{r}}$ contain weights; their $i$ th diagonal elements are $w_{i}^{(\mathrm{c})}$ and $\nu^{1 / 2}$, respectively.

Letting $\mathbf{A}$ be the product of the two matrices on the left side of Equation (22), we use singular value decomposition (SVD; e.g. Press and others, 1986, p. 52) to find a generalized inverse of $\mathbf{A}$, after zeroing the inverses of singular values that fail to pass a round-off criterion. In practice, with our smoothness regularization criterion (Equations (15) and (17)), singular values are only occasionally too small.

Iterations continue until changes in $\Delta \mathbf{p}^{(k)}$ are small or fail to reduce $I_{\mathrm{p}}$.

Second, even when Equation (13) has been satisfied, the vector $\mathbf{p}$ will produce model observables $\mathbf{o}^{(\mathrm{m})}$ (layer depth, ice-surface elevation, and mass balance or ice velocity at a number of points on the surface) that may not satisfy the mismatch constraint given by Equation (11). Therefore, the estimate of the trade-off parameter $\nu$ must also be improved by iteration, until Equation (11) is satisfied. Figure 1, based on application of the method to observations at Taylor Mouth

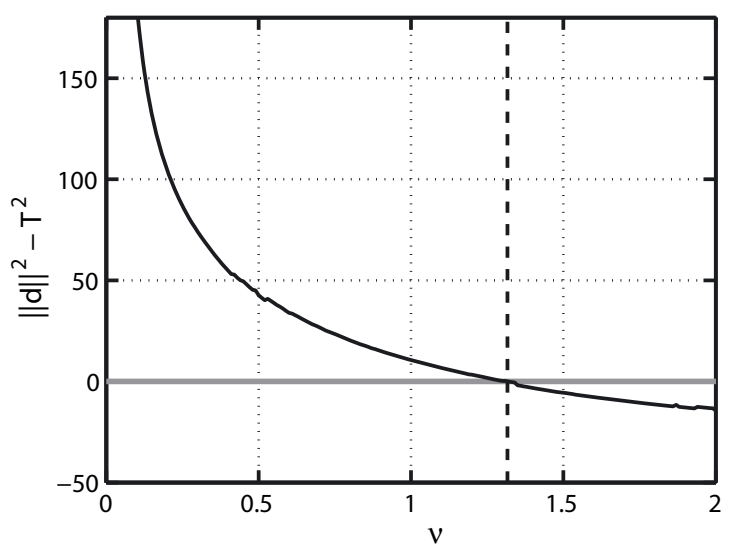

Fig. 1. Sensitivity of data-mismatch criterion $\left(\|\mathbf{d}\|^{2}-T^{2}\right)$ to tradeoff parameter, $\nu$. For each value of $\nu$, we find the model-parameter vector $\mathbf{p}$ that minimizes $I_{p}$. For data from Taylor Mouth (Figs 2-4), $\nu \simeq 1.3$ satisfies Equation (11), and we accept the corresponding solution vector $\mathbf{p}$.

(section 4), shows how the data mismatch $\|\mathbf{d}\|^{2}$ typically varies with trade-off parameter $\nu$. When $\nu$ is small, minimizing $I_{p}$ with respect to the parameters (Equation (13)) overemphasizes model smoothness at the expense of a large data misfit $\|\mathbf{d}\|^{2}$. When $\nu$ is large, minimizing $I_{p}$ overemphasizes data fit, making $\|\mathbf{d}\|^{2}$ very small at the expense of model smoothness, and $\left(\|\mathbf{d}\|^{2}-T^{2}\right)$ becomes negative; the data vector $\mathbf{d}$ (or the noise in the data) has been fit more closely than the data uncertainties can justify. This high degree of fit is achieved by the presence of extraneous structure in the solution vector $\mathbf{p}$. For Taylor Mouth data, as shown in Figure 1, the model fits the data at the expected level $T^{2}$ for $\nu=1.3$, and the model-parameter vector $\mathbf{p}$ found when using this value of $\nu$ is the preferred solution.

\section{TAYLOR MOUTH}

To illustrate the solution of an inverse problem with this approach, we have found the spatial accumulation-rate pattern at Taylor Mouth, a site in Antarctica at the head of Taylor Valley (Fig. 2). Taylor Mouth is approximately $30 \mathrm{~km}$ northeast of the Taylor Dome ice-core site, where an ice core to bedrock was collected in 1994 (Grootes and others, 1994). That core has provided a $150 \mathrm{kyr}$ stable-isotope paleoclimate record (Steig and others, 2000; Grootes and others, 2001) and an atmospheric $\mathrm{CO}_{2}$ record for the past $60 \mathrm{kyr}$ (Indermuhle and others, 2000). Several short (100 m) firn cores were also collected, in order to assess the spatial variability of climate, and climate-signal preservation in the area. One of these cores was collected from Taylor Mouth (Neumann and others, 2005).

\subsection{Observations and flowband}

The ice at the Taylor Mouth core site (TM in Fig. 3) originates along a flowline from a saddle approximately $12 \mathrm{~km}$ to the northwest (Figs 2 and 3). Strong internal layers are visible in the radar profile (Fig. 4). The strain network shown in Figure 3 provided surface ice-velocity data. We surveyed the flow markers by optical methods tied to a local bedrock monument (on the nunatak near [49 km, $10 \mathrm{~km}]$ in Fig. 3), which had been tied to a reference GPS base station at 


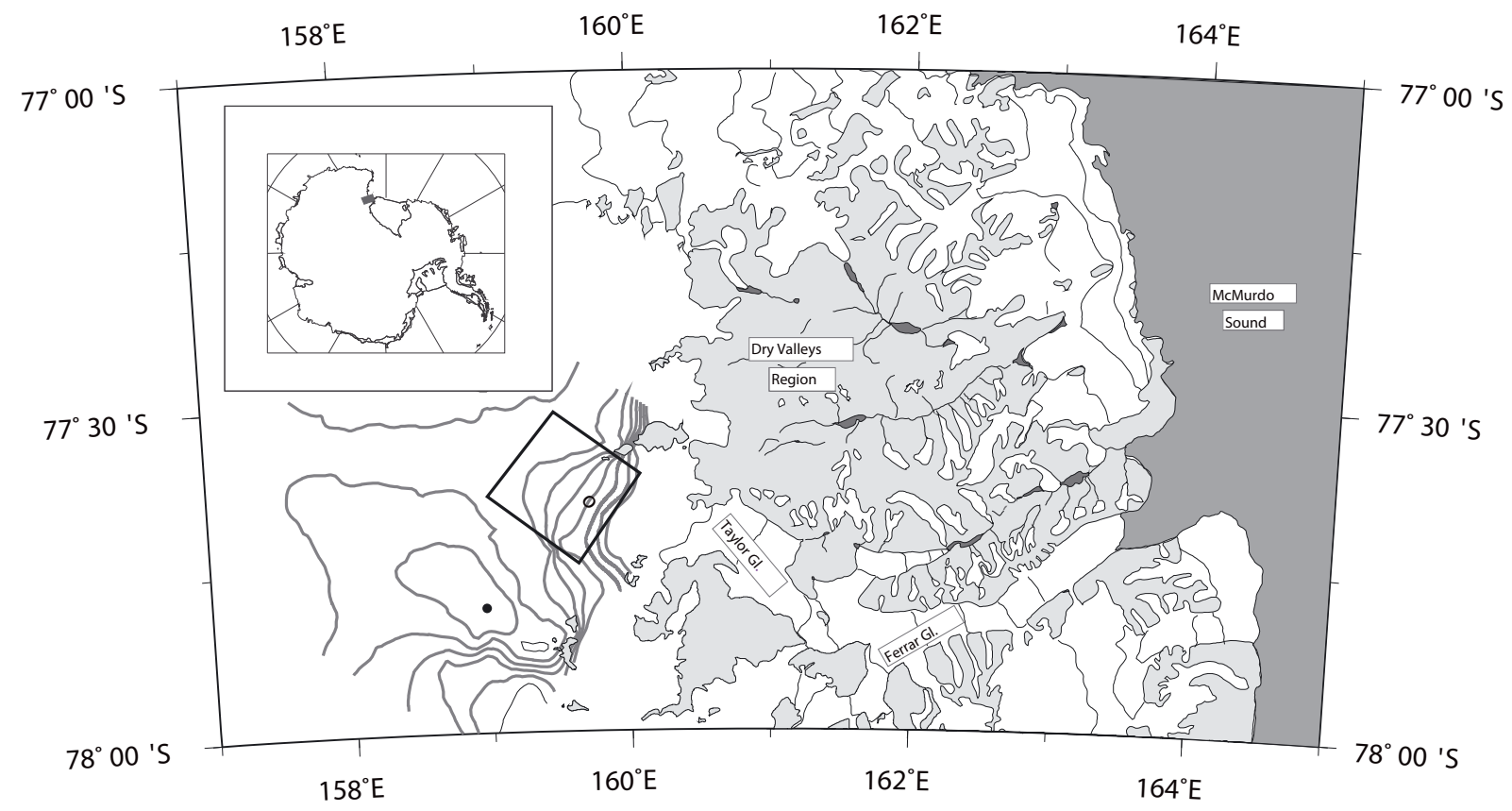

Fig. 2. Location of Taylor Dome. Solid dot marks location of $554 \mathrm{~m}$ Taylor Dome ice core. Surface-elevation contours were measured by airborne radar survey (Morse, 1997). Box denotes Taylor Mouth study site, shown in Figure 3. Open circle marks $100 \mathrm{~m}$ core.

McMurdo Station, Antarctica, as described by Morse and others (2007). We estimated the standard error of these velocity data to be $\sigma_{u}^{(\mathrm{d})}=0.05 \mathrm{~m} \mathrm{a}^{-1}$. To find the flowline shown in Figure 3, we interpolated the velocity field between these measured markers. Starting at the core site and working both upstream and downstream, we tracked the locus of points that was everywhere parallel to the local velocity field. To find the relative flowband width $W(x)$ shown in Figure $5 \mathrm{~d}$, we repeated the flowline calculation, starting from two points

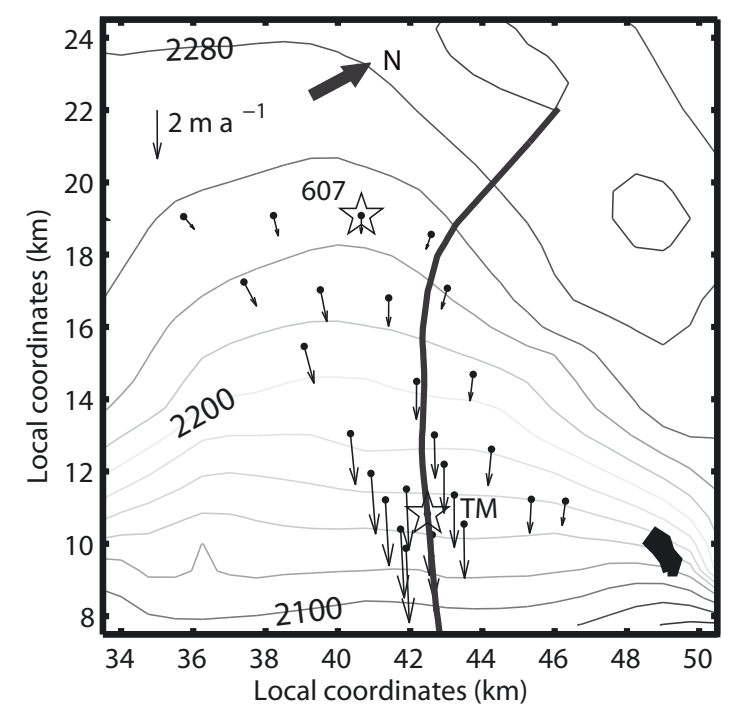

Fig. 3. Surface topography and ice flow of Taylor Mouth area. Local coordinate system is as described by Morse and others (2007). Dots represent poles in strain grid used to infer surface ice-flow velocities and the flowband through the core site at TM. Stars denote locations of gross- $\beta$ accumulation and $10 \mathrm{~m}$ firn-temperature measurements. Black area in lower right is a nunatak, to which velocity surveys were referenced. near the core site but offset several hundred meters in opposite directions transverse to the flow. The relative flowband width $W(x)$ is the separation between these two flowlines, normalized by their separation at the core site.

We measured ice thickness along that central flowline with the University of Washington ice-penetrating radar system described by Weertman (1993) and Gades (1998). This profile also includes internal layers (Fig. 4); we attribute a standard error of $\sigma_{h}^{(\mathrm{d})}=5 \mathrm{~m}$ to the depth of the layer indicated by the dashed curve. We converted the radar travel times to depths using the known source-receiver separation, and wave speeds of $300 \mathrm{~m} \mathrm{\mu s}^{-1}$ in air for the direct wave, and $168 \mathrm{~m} \mathrm{\mu s}^{-1}$ in ice. After accounting for the two-way travel time through the ice and approximately $15 \mathrm{~m}$ of air in the firn, we recovered ice-equivalent depths.

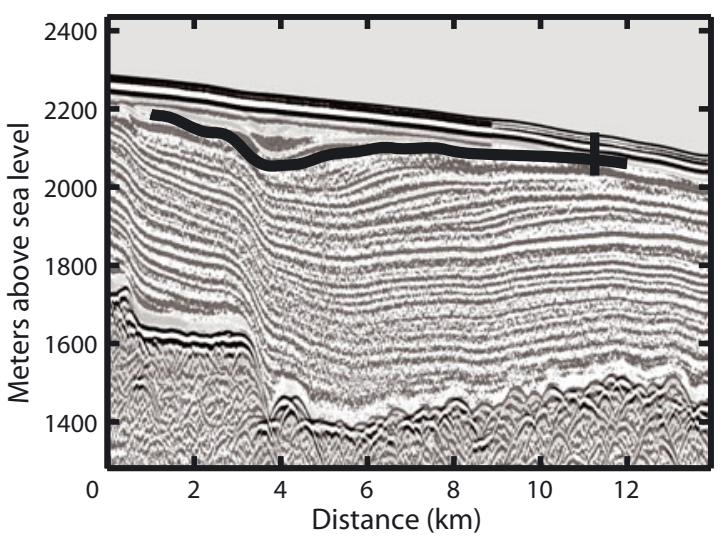

Fig. 4. Ice-penetrating radar profile along Taylor Mouth flowband. Vertical bar marks the $100 \mathrm{~m}$ core hole. Bold solid curve marks the internal layer used as data in the inverse problem. (Apparent surface-parallel features in upper $50 \mathrm{~m}$ are an artifact.) 


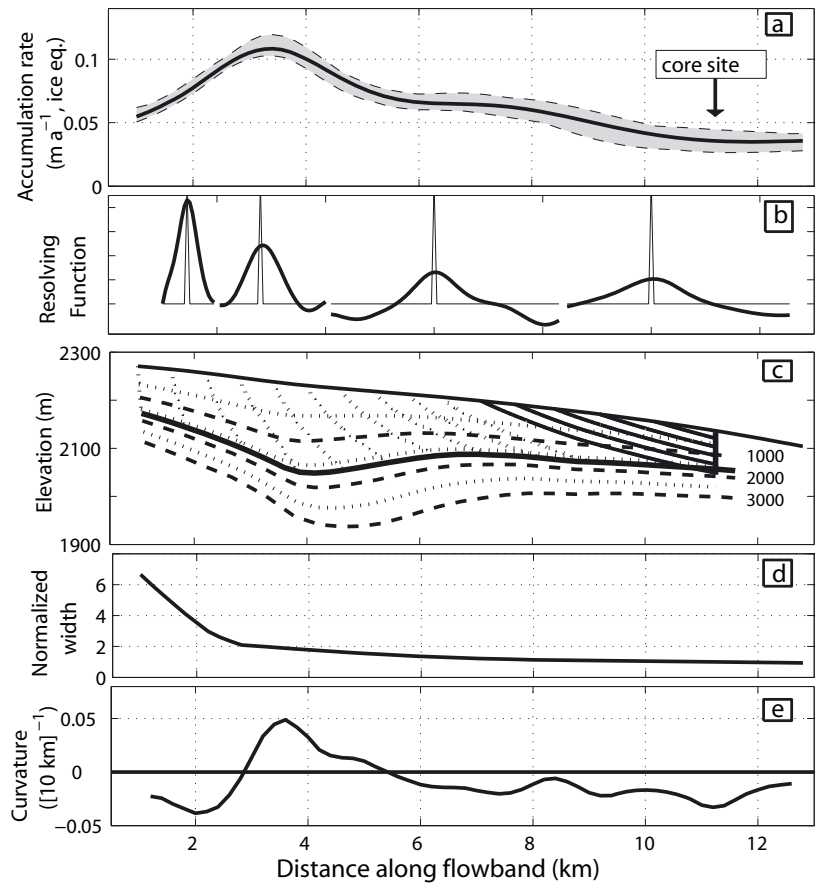

Fig. 5. (a) Accumulation-rate solution $\dot{b}(x)$. Gray band spans range of solutions associated with uncertainties in data as described in section 5.2. (b) Narrow triangles show locations and widths of a series of perturbations (amplitudes not to scale) that were added to individual nodes of the preferred accumulation-rate solution in (a). Each corresponding bold curve is a model-resolving function, which shows the ability of the inverse procedure to recover that perturbation. (c) Vertical section along flowband. End points of dotted particle paths define 1662 year layer (bold solid curve). Other modelled layers, at 500 year intervals, are shown by dashed and dotted subhorizontal curves. Other particle paths (solid curves) provide model ages for the ice core (bold vertical line). (d) Non-dimensional flowband width $W(x)$, inferred from surface topography and surfacevelocity measurements (Fig. 3). (e) Positive surface curvature (concave topography) correlates with high accumulation rate, probably due to deceleration of both upslope and downslope winds.

Surface elevations in Figure 3 were measured along $5 \mathrm{~km}$ gridlines as part of an airborne ice-penetrating radar survey with GPS control (Morse, 1997). We also measured the surface-elevation profile shown in Figure $5 c$, with a groundbased continuous GPS survey along the flowline. The surface elevations are known to within $\sigma_{S}^{(\mathrm{d})}=2 \mathrm{~m}$.

Morse and others (1999) detected the radioactive horizons from above-ground bomb testing in the 1950s and 1960s (Picciotto and Wilgain, 1963) using gross- $\beta$ measurements on a shallow core. As a result of the low net accumulation rate at the Taylor Mouth core site (Fig. 5), these horizons are not well preserved, resulting in relatively low confidence (high uncertainty) in the accumulation-rate measurement. The average accumulation rate at the core site was $\dot{b}=$ $0.023 \mathrm{~m} \mathrm{a}^{-1}$, with $\sigma_{\dot{b}}^{(\mathrm{d})}=0.01 \mathrm{~m} \mathrm{a}^{-1}$ over the four decades between 1955 and 1994.

\subsection{Data for inverse problem}

We used our inverse procedure to determine the accumulation rate at 60 nodes $x_{i}^{(\mathrm{n})}$ spaced at $200 \mathrm{~m}$ along the flowband through the Taylor Mouth core site. Our data comprised: the ice-surface profile (Fig. 5c); the uppermost continuous
Table 1. Expected values and solution characteristics for model parameters $p_{j}$ and trade-off parameter $\nu$. Ice flux $q_{\text {in }}$ is reported in $\mathrm{m}^{3} \mathrm{a}^{-1}$ per $\mathrm{m}$ width of flowband at the boundary. $H_{0}$ is the iceequivalent thickness at the core site. Standard deviations of the solution were calculated from 100 parameter solutions derived from randomly perturbed datasets

\begin{tabular}{|c|c|c|c|c|}
\hline Parameter & $\begin{array}{l}\text { Expected } \\
\text { value } p_{j}^{(\mathrm{e})}\end{array}$ & $\begin{array}{c}\text { Acceptable } \\
\text { deviation } \delta p_{j}^{(\mathrm{c})}\end{array}$ & $\begin{array}{l}\text { Solution } \\
\qquad p_{j}\end{array}$ & $\begin{array}{l}\text { Standard } \\
\text { deviation }\end{array}$ \\
\hline$\dot{b}_{j}$ & Fig. 6 & smooth & Fig. 6 & Fig. 5 \\
\hline$q_{\text {in }}$ & $16.5 \mathrm{~m}^{2} \mathrm{a}^{-1}$ & $3.8 \mathrm{~m}^{2} \mathrm{a}^{-1}$ & $17.8 \mathrm{~m}^{2} \mathrm{a}^{-1}$ & $2.2 \mathrm{~m}^{2} \mathrm{a}^{-1}$ \\
\hline $\mathcal{A}$ & 2000 years & 400 years & 1662 years & 126 years \\
\hline$H_{0}$ & $646.5 \mathrm{~m}$ & $2 \mathrm{~m}$ & $647.8 \mathrm{~m}$ & - \\
\hline$E$ & 1.0 & 0.3 & 0.75 & - \\
\hline \multirow[t]{2}{*}{$q_{\text {geo }}$} & $77 \mathrm{~mW} \mathrm{~m}^{-2}$ & $5 \mathrm{~mW} \mathrm{~m}^{-2}$ & - & - \\
\hline & $\nu^{(0)}$ & & Solution & \\
\hline$\nu$ & 1.0 & - & 1.3 & - \\
\hline
\end{tabular}

internal layer (highlighted in Fig. 4); the ice velocity measured at the core site and at three other sites along the flowband (see Fig. 3); and the accumulation rate measured at the core site (TM in Fig. 3). The ice-surface data comprised elevations at the $N_{S}=60$ nodes $x_{i}^{(\mathrm{n})}$ spaced at $200 \mathrm{~m}$, and the internal-layer data comprised measured depths at the ending $x$ positions of 70 particle paths in the forward model (e.g. see Fig. 5c).

\subsection{Site-specific simplifications}

In section 3, we described the inversion procedure in very general form. We now select an appropriate thermomechanical ice-flow model for the Taylor Mouth flowband. This flow model should be as sophisticated as necessary to accurately represent the flow field as it impacts the targeted layer along this flowband, while being as simple as possible to facilitate the inversion procedure.

\section{Thermomechanical calculations}

At Taylor Mouth, we use horizontal-velocity shape functions $\phi(x, \hat{z})$ based on the SIA (e.g. Paterson, 1994, p. 262), incorporating depth-varying temperature. Measured accumulation rates are only a few centimeters (ice equivalent) per year, so vertical velocities are small and advective heat transfer is unimportant. We calculate a 1-D vertical temperature profile $\theta(x, z)$ at each node $x_{i}^{(\mathrm{n})}$ using the 1-D steadystate temperature model of Firestone and others (1990). By using the Taylor Dome value of geothermal flux (Table 1), in which we have high confidence, we decouple $q_{\text {geo }}$ from the other model parameters. Since the basal temperature is below the melting point everywhere along the flowband, we set basal sliding $u(x, 0)=0$ and basal melting $\dot{m}(x)=0$, further simplifying the inverse problem. Details of our thermomechanical ice-flow calculations are provided in Appendix B.

\section{Jacobian matrices}

The matrices $\mathbf{J}_{\mathrm{c}}$ and $\mathbf{J}_{\mathrm{r}}$ of partial derivatives $\partial c_{i} / \partial p_{j}$ and $\partial r_{i} / \partial p_{j}$ in Equation (22) are straightforward to construct numerically, by perturbing each parameter $p_{j}$ in turn, and recalculating all the model residuals $c_{i}$ and data residuals $r_{i}$ with 

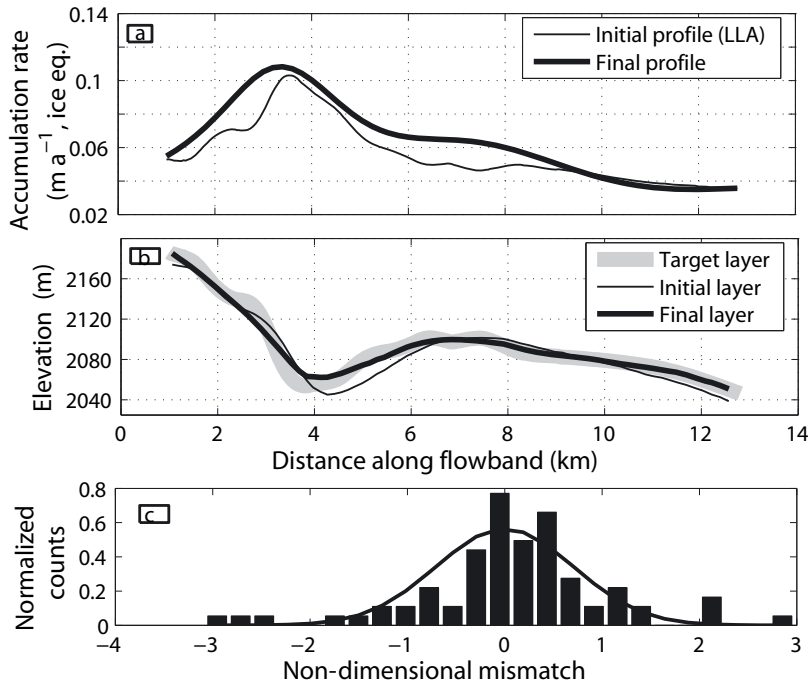

Fig. 6. (a) Model estimates of $\dot{b}(x)$ start from the LLA (thin solid curve), and converge to bold solid curve. (b) Corresponding modelled internal-layer shapes. Initial estimate (thin solid curve), found by using LLA-derived accumulation in (a) in forward algorithm, is a poor match to the observed layer (solid gray curve). Final modelled layer is shown by bold solid curve. (c) Distribution of nondimensional mismatches (Equation (18)) between observations and model, normalized to have unit integral. Resemblance to normal probability-density distribution (solid curve) confirms that Equation (20) defines appropriate tolerance level $T$.

the forward thermomechanical model. However, this procedure is computationally intensive. Instead, we first note that the expressions for $c_{i}$ in Equations (15) and (16) can be differentiated directly. Then, noting that the residuals $r_{i}$ (Equation (18)) depend on the parameters $p_{j}$ only through the observables $o_{i}^{(\mathrm{m})}$, we express each observable quantity $o_{i}^{(\mathrm{m})}$ as a function of the model parameters $\mathbf{p}$ so that we can find analytical expressions for these derivatives wherever possible. For example, the accumulation rate $\dot{b}(x)$ at any $x$ can be interpolated from the $b_{i}$ model parameters. Ice flux $q(x)$ can be related to $\mathbf{p}$ through Equation (2). Velocity $u(x, S(x))$ at the ice surface $S(x)$ at any position $x$ can be expressed in terms of the parameters by first expressing velocity in terms of flux $q(x)$ using Equations (3) and (4).

\section{Surface-profile data}

The modelled ice-surface profile $S^{(\mathrm{m})}(x)$ cannot be written readily as an analytical function of the model parameters, and as a result, the partial derivatives $\partial S^{(\mathrm{m})}\left(x_{i}^{(\mathrm{n})}\right) / \partial p_{j}$ need to be evaluated numerically. We avoided this computational cost by decoupling the ice-surface residuals from the other data, and at the same time decoupling the enhancement factor, $E$, from the other model parameters. $E$ appears only in Equation (B8) (see Appendix B), where it is used to find the steady-state surface profile, $S^{(\mathrm{m})}(x)$, with a dynamic calculation. We chose to use the observed surface, $S^{(\mathrm{d})}(x)$, while finding values for all the other parameters, $\mathbf{p}$, in the inversion process. After solving the inverse problem, we incorporated those solution parameters in Equation (B8), and adjusted $E$ to minimize the mismatch between the modelled surface, $S^{(\mathrm{m})}(x)$, and the observed surface, $S^{(\mathrm{d})}(x)$. For our Taylor Mouth dataset, we were able to match the surface to within observational error, using $E=0.75$. This internal consistency after the calculations were completed justifies decoupling $E$ from the other model parameters.

For some other datasets, it may be more important to include $E$ as a coupled model parameter throughout the inversion, and to include the observed surface topography, $S^{(\mathrm{d})}(x)$, as data to be matched at each iteration.

\section{Model-parameter considerations}

For this inverse solution, we assigned a characteristic length scale, $L^{(\mathrm{c})}=700 \mathrm{~m}$, which is comparable to the ice thickness, and a characteristic accumulation rate, $\dot{b}^{(\mathrm{c})}=0.025 \mathrm{~m} \mathrm{a}^{-1}$, which is comparable to the value at the core site. These values were used in Equation (15). We initially estimated the input flux, $q_{\text {in, }}$ by extrapolating the flowband upstream to the ice divide, and applying $\dot{b}^{\text {(c) }}$ in that area. We initially estimated the layer age with the LLA, using the measured depth of the layer at the core site, and the average of $\dot{b}^{\text {(c) }}$ and the measured accumulation rate at the core site. We attributed large acceptable deviations, $\delta p_{j}^{(\mathrm{c})}$, to these model parameters (see Equation (16), and Table 1). As a result, our preconceptions had only a small impact on the solution for $q_{\text {in, }}$ and $\mathcal{A}$. In Table 1 we show expected values, $p_{j}^{(\mathrm{e})}$, and acceptable deviations, $\delta p_{i}^{(\mathrm{c})}$, from those expectations for the model parameters $q_{\text {in }}, \mathcal{A}, H_{0}, E$ and $q_{\text {geo }}$. Our site-specific simplifications allowed us to decouple $H_{0}, E$ and $q_{\text {geo }}$ from the main inverse problem, and thereby to significantly reduce computational time. In section 5.1, we show why this simplification was justified.

\section{RESULTS}

\subsection{Model parameters and predicted observables}

We solved the non-linear system of Equation (13) for the range of values of $\nu$ shown in Figure 1 . The mismatch criterion Equation (11) is satisfied by $\nu=1.3$; we accepted the accumulation-rate profile and other model parameters associated with $\nu=1.3$.

Using the preferred value for the trade-off parameter, $\nu=$ 1.3, we inferred the accumulation-rate profile in Figure 6a, shown before and after it evolved through successive iterations. The corresponding modelled internal-layer shapes are shown in Figure 6b. The final modelled layer agrees closely (but not too closely) with the observed layer. Table 2 shows how well the modelled observables match the data. In spite of the large observational uncertainty, the modelled accumulation rate at the core site exceeds the measured value from bomb fallout by more than $1 \sigma_{\dot{b}}^{\text {(d) }}$. Three of the four modelled surface velocities matched the measured velocities to within $1 \sigma_{u}^{(d)}$. To estimate the standard deviations of the modelled observables in Table 2, we created 100 predictions of all observables using 100 realizations of the parameter solution, as described in section 5.2.

Figure $6 \mathrm{c}$ shows a histogram of the mismatches $(70$ points in the layer-depth profile, four surface velocity points, and accumulation at the core site), non-dimensionalized with the standard errors of the data. The histogram amplitudes are also normalized so that they integrate to unity. Because we incorporated the mismatch criterion Equation (11), the mismatches do approximate a set of samples from a probabilitydensity distribution with zero mean and unit variance (thin solid curve in Fig. 6c), i.e. the model solution is a reasonable fit to the data. 
The solution of the inverse problem also returned the age of the layer, $\mathcal{A}=1662$ years, and the ice flux entering the upstream end of the flowband, $q_{\text {in }}=17.8 \mathrm{~m}^{3} \mathrm{a}^{-1}$ per meter width of flowband. To assess the appropriateness of decoupling $H_{0}$ and $E$ from the other model parameters, we ran a series of dynamic steady-state forward models as described by Equation (B8) in Appendix B, and driven by our preferred solution for boundary flux, $q_{\text {in, }}$ and accumulation-rate profile, $\dot{b}(x)$. We varied the ice thickness, $H_{0}$, at the core site and enhancement factor, $E$, in Equation (1), and for each pair we calculated the root-mean-square $(\mathrm{rms})$ mismatch between the surface-profile data and the surface predicted by the forward calculation. Figure 7 shows that the surface profile from the dynamic flow model agreed with the measured surface to within $1 \mathrm{~m}$ (rms) for $H_{0}=647 \mathrm{~m}$ and $E=0.75$. This finding confirms that if we had included $H_{0}$ and $E$ as model parameters in the general inverse procedure, we would have obtained $E \approx 1$, in agreement with our expectations, and the other model parameters, $\mathbf{p}$, would have been very similar to those that we actually obtained. Therefore, decoupling the surface topography calculation and the enhancement factor from the inverse problem was appropriate at Taylor Mouth.

\subsection{Confidence limits on solution}

Because of the standard errors associated with each observation, our dataset is just one realization out of an infinite number of possible realizations of the same set of observable quantities. In order to estimate uncertainties in the derived model-parameter vector $\mathbf{p}$, we created 100 synthetic datasets, and derived a corresponding set of model parameters from each. We assumed that each observation $o_{j}^{(\mathrm{d})}$ of accumulation rate or velocity was independent of the other data. In each synthetic dataset, we replaced the accumulation-rate measurement and each velocity observation by a value selected at random from our estimate of its underlying normal probability-density distribution, defined by its mean (assumed to be its observed value $o_{j}^{(\mathrm{d})}$ ) and its observational uncertainty $\sigma_{j}^{(\mathrm{d})}$. We assumed that the observations of layer depth $h_{i}^{(\mathrm{d})}$ were cross-correlated. We represented correlated noise among the layer-depth data using a red-noise process. A red-noise series $f(x)$ can be generated by

$$
f(x+\Delta x)=\alpha f(x)+\sigma n(x),
$$

where $\alpha$ describes the cross-correlation of the noise series at lag $\Delta x$, and $\sigma$ is an amplitude scaling a random-noise series $n(x)$, with zero mean and unit variance. To create each

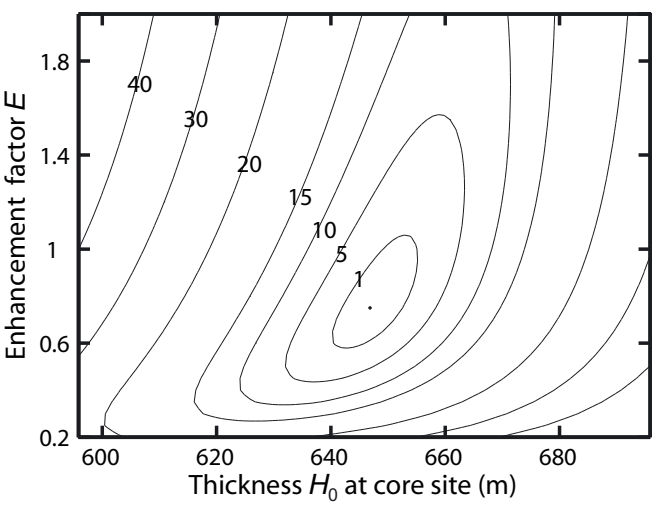

Fig. 7. Root-mean-square mismatch between observed surface topography and topography calculated with forward model (see Equation (B8) in Appendix B), for a range of ice thickness $H_{0}$ at core site, and enhancement factor $E$, using $q_{\text {in }}$ and $\dot{b}_{j}$ parameters from preferred solution of the inverse problem in all cases. Minimum at $H_{0}=646 \mathrm{~m}$ and $E=0.75$ suggests that if $H_{0}$ and $E$ had not been decoupled from the other parameters to simplify this particular inverse problem, values similar to these would have been obtained.

synthetic dataset, we added red noise to the observed layerdepth profile $h^{(\mathrm{d})}(x)$. We generated 100 red-noise series with Equation (23), using $\Delta x=200 \mathrm{~m}$ (the node separation) and $\alpha=0.9866$, which creates cross-correlation 'memory' over distances of several kilometers. We used $\sigma=5 \mathrm{~m}$, which is also the uncertainty $\sigma_{h}^{(d)}$ in the layer-depth measurements. After solving the inverse problem for each of these 100 synthetic datasets, we constructed a statistical representation of the solution, by finding the mean and standard deviation of each parameter $p_{j}$.

The gray band in Figure 5a shows our estimate of the uncertainty in our accumulation-rate solution, based on the spread of results from this suite of experiments with synthetic datasets. Clearly, the peak in accumulation rate near $x=4 \mathrm{~km}$ is a robust feature. The associated standard deviations in layer ages $\mathcal{A}$ and incoming ice flux $q_{\text {in }}$ are shown in Table 1.

\subsection{Topography and accumulation rate}

King and others (2004) related accumulation-rate variations (at the scale of $1 \mathrm{~km}$ ) to modelled wind speed and snow transport associated with subtle slope changes at Lyddan ice rise, Antarctica. Vaughan and others (1999) found a strong

Table 2. Data and modelled observables. Standard deviations of modelled observables were calculated from 100 sets of data predictions using 100 parameter solutions derived from randomly perturbed datasets

\begin{tabular}{|c|c|c|c|c|}
\hline Datum & $\begin{array}{l}\text { Measured } \\
\text { value } o_{i}^{(\mathrm{d})}\end{array}$ & $\begin{array}{c}\text { Measurement } \\
\text { uncertainty } \sigma_{j}^{(\mathrm{d})}\end{array}$ & $\begin{array}{l}\text { Modelled } \\
\text { value } o_{i}^{(\mathrm{m})}\end{array}$ & $\begin{array}{c}\text { Standard } \\
\text { deviation } \sigma_{j}^{(\mathrm{m})}\end{array}$ \\
\hline$\dot{b}_{1}$ (core site) & $0.023 \mathrm{~m} \mathrm{a}^{-1}$ & $0.010 \mathrm{~m} \mathrm{a}^{-1}$ & $0.036 \mathrm{ma}^{-1}$ & $0.009 \mathrm{~m} \mathrm{a}^{-1}$ \\
\hline$\dot{b}_{2}($ site 607$)$ & $0.051 \mathrm{~m} \mathrm{a}^{-1}$ & $0.005 \mathrm{~m} \mathrm{a}^{-1}$ & $0.070 \mathrm{ma}^{-1}$ & $0.006 \mathrm{ma}^{-1}$ \\
\hline$u_{1}$ & $0.69 \mathrm{~m} \mathrm{a}^{-1}$ & $0.05 \mathrm{~m} \mathrm{a}^{-1}$ & $0.73 \mathrm{~m} \mathrm{a}^{-1}$ & $0.016 \mathrm{ma}^{-1}$ \\
\hline$u_{3}$ & $2.65 \mathrm{~m} \mathrm{a}^{-1}$ & $0.05 \mathrm{~m} \mathrm{a}^{-1}$ & $2.59 \mathrm{~m} \mathrm{a}^{-1}$ & $0.029 \mathrm{~m} \mathrm{a}^{-1}$ \\
\hline$u_{4}$ & $2.81 \mathrm{~m} \mathrm{a}^{-1}$ & $0.05 \mathrm{~m} \mathrm{a}^{-1}$ & $2.80 \mathrm{~m} \mathrm{a}^{-1}$ & $0.031 \mathrm{~m} \mathrm{a}^{-1}$ \\
\hline
\end{tabular}




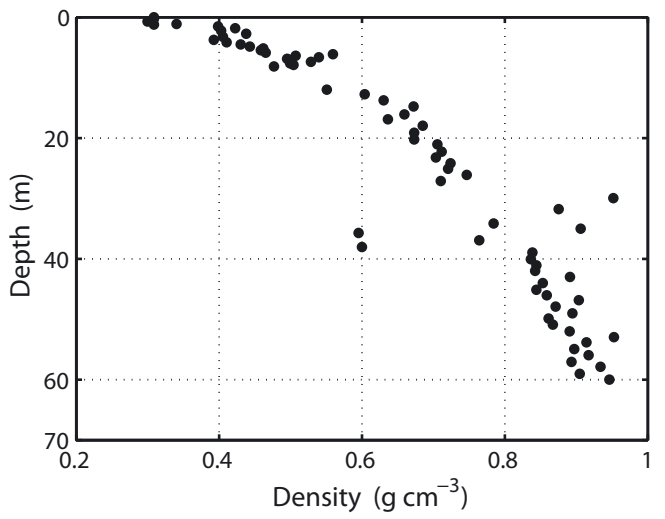

Fig. 8. Density variation with depth in the Taylor Mouth core, based on mass and volume measurements of core sections (data from P.M. Grootes and E.J. Steig).

correlation between accumulation rate and surface slope $\mathrm{d} S / \mathrm{d} x$ on Fletcher Promontory, Antarctica, and Frezzotti and others $(2004,2007)$ also found a correlation between net accumulation rate and surface slope in East Antarctica. At Taylor Mouth, accumulation is also related to topography; however, we find a better correlation with surface curvature $d^{2} S / d x^{2}$. Highest accumulation occurs where the surface is concave (near $x=4 \mathrm{~km}$ in Fig. 5e). There, upslope winds encounter steepening slopes, and downslope winds encounter lessening slopes. In both cases, wind speed tends to decrease, and suspended and saltating snow tends to be deposited. This effect is probably widespread; Leonard and others (2004) found a region of high accumulation rate along the western shore of Subglacial Vostok Lake, Antarctica, where the surface is also concave upward.

\subsection{Depth-age scale}

Since the Taylor Mouth core is $100 \mathrm{~m}$ long, it is composed primarily of firn rather than ice. Because the flowband model uses ice-equivalent thickness, we converted the measured thickness along the flowband to ice-equivalent thickness $H(x)$ using the density-depth profile $\rho(z)$ (Fig. 8), measured on the Taylor Mouth ice core. Near-surface density at the core site was also measured by M. Duval (personal communication) in a $2 \mathrm{~m}$ deep snow pit.

We then used our accumulation-rate solution (Fig. 5a), together with the particle-tracking module described in section 2, to calculate the steady-state depth-age relationship (in ice equivalent) of the Taylor Mouth ice core. This depthage estimate takes into account spatial variations in accumulation rate, ice thickness, and strain associated with flow upstream from the core site. Finally, we converted this depthage relation back to true depth, using $\rho(z)$. This depth-age scale is shown by the solid white curve in Figure 9. The gray band shows the range of depth-age curves resulting from the range of solutions, $\mathbf{p}$, that generated the gray band in Figure 5a.

\section{DISCUSSION}

\subsection{Steady-state assumption}

We assumed that ice flow at Taylor Mouth is in steady state. We used a time-dependent flowband model (not described here) to verify this assumption. Following a climate perturbation, ice flux in our time-dependent flow model relaxed with an e-folding time of approximately 5000 years. The climate at Taylor Dome has probably been relatively stable over the past 6000 years (Morse and others, 1998; Steig and others, 1998; Monnin and others, 2004), so iceage transients have largely run their course, and therefore ice flow at Taylor Mouth is approximately in steady state.

Our steady-state forward ice-flow model, when run with the accumulation-rate solution (Fig. 5a), also matches the shapes of deeper layers, although, as might be expected, with a somewhat higher standard deviation $(\sim 10 \mathrm{~m})$ than we find for the layer that we used in the inverse problem. We also obtained solutions for (assumed) steady accumulationrate profiles by repeating the inversion process, using several successively deeper layers with ages up to 2700 years in the solutions. These accumulation-rate solutions had magnitudes and structure similar to the solution in Figure 5a. These results suggest that our assumption of steady-state thickness and flow is reasonable, over millennial timescales.

\subsection{Millennial accumulation patterns}

Accumulation rates derived from near-surface internal layers by the SLA are necessarily short-term averages (e.g. 10100 year averages). Since accumulation rates in the polar regions are influenced by multi-annual (El Niño-Southern Oscillation (ENSO), e.g. Bromwich and others, 2004) to multi-decadal atmospheric cycles (Arctic/Antarctic Oscillations, e.g. Appenzeller and others, 1998; Noone and Simmonds, 2002), short-term accumulation rates may not be applicable over longer timescales, and should be used with caution in ice-flow studies. Our formal inverse-problem approach allows deeper (and therefore older) internal layers to be used to determine longer-term average accumulation rates.

Our geophysical-inverse approach assumes that accumulation rate is constant in time, and that ice flow is steady. For deep layers that pre-date the relative stability of the late Holocene, these may be questionable assumptions. It should be possible to relax these two model assumptions independently. We could determine the accumulation-rate pattern at different times in the past by using multiple layers in the inverse procedure. In addition, replacing Equation (B8) with a transient continuity equation (e.g. Paterson, 1994, p. 322) would also allow for changes in ice thickness along the flowband as a function of time.

\subsection{Response to incompatible data}

The inversion procedure assumes steady-state accumulation and flow, and expects the data to represent long-term average values of the observable quantities. Ice-sheet thickness and velocity change slowly, and are insensitive to decadal or centennial fluctuations in accumulation rate, because of the 5000 year response time. It takes a long time for accumulation variations to build up thicker ice or steeper slopes, and these slow changes control velocity changes. Although we obtained our velocity and ice-thickness data over a period of only a few years, we can expect those data to be representative of behavior on a millennial timescale.

In contrast, accumulation rate can change rapidly (Van der Veen, 1993; McConnell and others, 2000). The inversion procedure can inform us how well observations represent long-term averages. One of the larger mismatches $(\approx 1.5 \sigma)$ among all our data points (see Table 2) occurred for the measured accumulation rate at the Taylor Mouth core 
site. The measured value $\left(0.023 \pm 0.01 \mathrm{~m} \mathrm{a}^{-1}\right)$, using bomb radioactivity, represents an average over approximately 40 years. This value is less than two-thirds of the solution of $0.036 \pm 0.009 \mathrm{~m} \mathrm{a}^{-1}$ in Figure $5 \mathrm{a}$, which provides an average value over 1662 years.

This large mismatch suggests that the accumulation rate between 1955 and 1995 was significantly lower than the millennial average. Morse and others (1999) used gross- $\beta$ activity in bomb layers to measure accumulation rate at a second site (607 in Fig. 3). Because site 607 lies $2 \mathrm{~km}$ from the flowline, we did not use its accumulation-rate data $(0.051 \pm$ $0.005 \mathrm{~m} \mathrm{a}^{-1}$ ) when solving the inverse problem. However, the 'bowl' shape of the Taylor Mouth catchment (Fig. 3) tends to funnel katabatic flow, so it is reasonable to expect that accumulation rate at any given time varies primarily in the downslope direction, and is relatively uniform along the contours over short distances where the slopes do not vary significantly. Site 607 projects along the $2250 \mathrm{~m}$ contour onto the flowband at $x=5.5 \mathrm{~km}$, where our solution (Fig. 5a) finds a larger accumulation rate of $0.070 \pm 0.006 \mathrm{~m} \mathrm{a}^{-1}$.

At both site 607 and the core site, the millennial value of accumulation rate is approximately $50 \%$ larger than the measurements from 1955 to 1994, indicating that the late 20th century was probably relatively dry throughout the Taylor Mouth area.

Measurements of cosmogenic ${ }^{10} \mathrm{Be}$ also suggest that the accumulation rate derived from bomb layers was lower than the long-term average. Over timescales longer than the 11 year sunspot cycle, ${ }^{10} \mathrm{Be}$ is often assumed to fall out at a constant rate. Because snow dilutes the ${ }^{10} \mathrm{Be}$, the concentration of ${ }^{10} \mathrm{Be}$ should be inversely proportional to the accumulation rate. Steig (1996) measured the average concentration of ${ }^{10} \mathrm{Be}$ at Taylor Mouth in the upper $5 \mathrm{~m}$, which spans approximately 80 years. On the basis of those measurements, Morse and others (1999) reported an average accumulation rate of $0.0395 \mathrm{~m} \mathrm{a}^{-1}$ at the Taylor Mouth core site. This value is indistinguishable from our millennial average of $0.036 \pm 0.004 \mathrm{~m} \mathrm{a}^{-1}$, suggesting that, if the late 20th century was dry in the Taylor Mouth basin, then the early 20th century had compensating above-average snowfall.

While technically reliable, the gross- $\beta$ measurement of accumulation rate at the Taylor Mouth core site was not a good measure of the quantity that the inversion procedure expected it to represent, i.e. an accumulation rate over the past 1662 years. Because of our steady-state assumption and the inherent short-term variability of accumulation, this data point was incompatible with the other data, which better represented conditions averaged over a millennium. As a result of this incompatibility, the inversion procedure was unable to fit this data point closely. Baldwin and others (2003) also noted systematic differences between short-term surface measurements of accumulation rate, and long-term values inferred from internal layers in Greenland.

\subsection{Resolving power}

Before making physical inferences from our preferred solution, it is important to assess the resolving power of the model. The model size is defined in section 3.3 through a smoothness condition on the accumulation-rate profile (Equation (15)). As outlined in section 3.1, this smoothness condition regularizes the model, and prevents spurious detailed structures from appearing in the model solution. It can also limit our ability to resolve real structures at fine spatial scales. Parker (1994) showed that, in a regularized model,

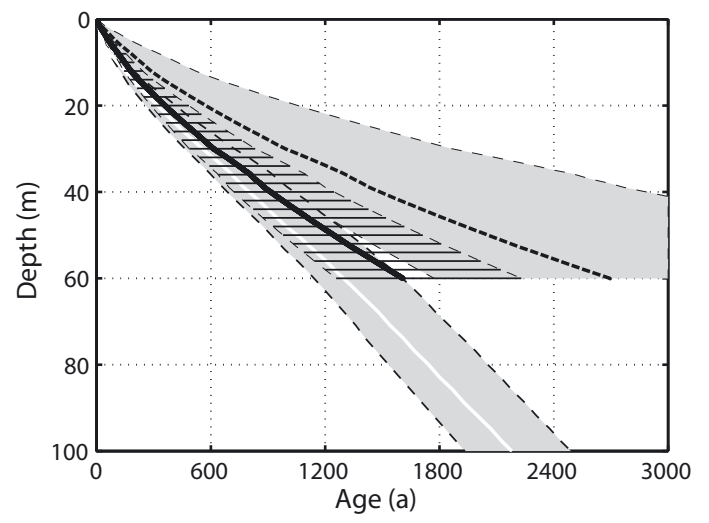

Fig. 9. Estimates of Taylor Mouth depth-age scale. Bold dashed curve produced by 1-D flow algorithm (Equation (35)), using measured density of the core (Fig. 8), and measured accumulation rate $\left(\dot{b}=0.023 \pm 0.010 \mathrm{~m} \mathrm{a}^{-1}\right)$ at the core site $(x=11.2 \mathrm{~km}$ in Fig. 5$)$. Gray region reflects uncertainty in measured $\dot{b}$. Solid black curve and hatched area result from 1-D flow algorithm (section 6.6) using long-term accumulation rate at the core site $\left(0.036 \pm 0.009 \mathrm{~m} \mathrm{a}^{-1}\right)$ inferred from flowband inverse problem. Solid white curve is derived from accumulation-rate solution and particle paths in Figure 5. Gray band around it spans age predictions from the same 100 accumulation-rate profiles used to generate gray band in Figure $5 \mathrm{a}$.

the true solution has been smoothed by a set of narrowly peaked resolving functions. These model-resolving functions indicate how closely the preferred solution is able to reproduce detailed structure in the underlying 'true' solution.

Following Parker (1994) and Truffer (2004), we can calculate the model-resolving function for each model parameter in our preferred solution, which we will call $\mathbf{p}_{0}$. Using $\mathbf{p}_{0}$ in the forward algorithm produces a synthetic dataset $\mathbf{o}_{0}^{(\mathrm{m})}$. Because the solution procedure does not fit the data exactly (see Equation (11)), this dataset $\mathbf{o}_{0}^{(\mathrm{m})}$ and the actual measured dataset $\mathbf{o}^{(\mathrm{d})}$ can be viewed as two equivalent realizations of the same underlying observables, and their differences $\mathbf{r}$ (see Equation (18)) are distributed across all dimensions of the data space. If we then solve the inverse problem using $\mathbf{o}_{0}^{(\mathrm{m})}$ as the data, we recover a new set of model parameters $\boldsymbol{p}_{0}^{\text {(rec) }}$ ('rec' for recovered). Because $\mathbf{p}_{0}$ and $\mathbf{p}_{0}^{(\text {rec })}$ are recovered from equivalent realizations of the data, their difference $\left(\mathbf{p}_{0}^{(\text {rec })}-\mathbf{p}_{0}\right)$ should be small, and distributed across all the model parameters. Their rms difference shows the level of uncertainty that we can expect in general in the solution.

Next we perturb in turn each model parameter $p_{j}$ in the preferred solution $\mathbf{p}_{0}$, producing a perturbed vector of model parameters that we call $\mathbf{p}_{(j)}$. We then run the forward model using each $\mathbf{p}_{(j)}$ to generate a perturbed set of observables $\mathbf{o}_{(j)}^{(\mathrm{m})}$. Using these calculated observables as new data $\mathbf{o}_{(j)}^{(\mathrm{d})}$, we then solve the new inverse problem to recover a new vector of parameters $\mathbf{p}_{(j)}^{(\text {rec })}$, using the trade-off parameter $\nu$ from the preferred model solution. The question of interest is how well we were able to recover the original perturbation to parameter $p_{j}$.

We cannot just subtract each new solution $\mathbf{p}_{(j)}^{(\mathrm{rec})}$ from our original preferred (and unperturbed) solution $\mathbf{p}_{0}$, because the small random differences $\left(\mathbf{p}_{0}^{(\mathrm{rec})}-\mathbf{p}_{0}\right)$ would confound the quest to isolate the recovered image of the perturbation. We eliminate that confounding effect by forming the difference 
$\left(\mathbf{p}_{(j)}^{(\text {rec })}-\mathbf{p}_{0}^{(\text {rec })}\right)$ to produce an image of the original impulsive perturbation to the corresponding model parameter $p_{j}$. This image is the model-resolving function for that model parameter. We would like to be able to recover that impulsive perturbation exactly; invariably, however, some information is lost. In general, the resolving functions also have non-zero amplitudes at other parameters. When the parameter $p_{j}$ is part of a spatial sequence (e.g. accumulation-rate parameters $\left.\dot{b}_{j}\right)$, its resolving function tends to display a broadened peak involving other nearby model parameters. The width of each resolving function gives a physical scale of meaningful structure that can be resolved in the inverse problem. Steep gradients in true accumulation rates will appear as spread-out gentler gradients if they are limited to length scales shorter than the width of the resolving function.

Narrow triangles in Figure 5b show perturbations added to individual nodes of the preferred accumulation-rate solution in Figure 5a. The corresponding bold curves are the model-resolving functions, which demonstrate the ability of the inverse procedure to recover details about accumulation rate at a single node. The width of the resolving function increases with distance along the flowband, because ice velocity increases with distance from the divide, and ice particles that comprise the target layer travel longer distances and can experience larger ranges of accumulation rate and strain. This indicates that further from the divide a layer retains less information about short-scale spatial variability in accumulation; therefore our ability to discern such spatial variability decreases with distance from the divide. However, our preferred accumulation-rate solution shows major variations on spatial scales larger than the half-width spread of the resolving functions (of $1-2 \mathrm{~km}$ ). Although only weighted mean values of accumulation rate can be resolved on length scales shorter than the resolving functions, the variations in our solution accurately reflect real trends over length scales of several kilometers.

Similarly, we can create data-resolving functions, by perturbing each data value in $\mathbf{o}^{(\mathrm{d})}$ in turn, to create new datasets $\mathbf{o}_{\text {pert }}^{(\mathrm{d})}$. Then we solve the inverse problem using each new dataset $\mathbf{o}_{\text {pert }}^{(\mathrm{d})}$ in turn to find new model-parameter solutions p. Finally, we run the forward model with each new parameter set $\mathbf{p}$ in turn, to generate associated sets of observables $\mathbf{o}^{(\mathrm{m})}$. Subtracting each new set of observables $\mathbf{o}^{(\mathrm{m})}$ from our original (and unperturbed) dataset $\mathbf{o}^{(\mathrm{d})}$ produces an image of the impulsive perturbation to the corresponding data value; this image is the data-resolving function for that observation. Each data-resolving function represents the degree to which that individual observable (data value) contributes unique information to the inverse procedure. Our data-resolution functions have widths comparable to those of the modelresolution functions. They also broaden in the downstream direction, indicating that useful information for the inverse problem is provided by progressively broader weighted averages of layer depth.

\subsection{Strain regimes and choice of forward algorithm}

Before attempting to extract accumulation-rate information from a particular layer, we must determine the appropriate level of complexity required in the thermomechanical forward algorithm. For any layer, we can distinguish three situations, based on the impact of vertical strain on the depth of the layer, and on the impact of gradients of strain and accumulation rate along particle paths that reach the layer.

\section{Shallow-layer approximation (SLA)}

For accumulation rate $\dot{b}$ and ice thickness $H$, a characteristic vertical strain rate is given by $\dot{\epsilon}_{\text {char }}=-\dot{b} / H$. After we account for firn compaction, very shallow layers with depth $h$ and age $\mathcal{A}$ have experienced almost no cumulative dynamic strain $\epsilon$ associated with ice flow, if

$$
|\epsilon| \approx\left|\dot{\epsilon}_{\text {char }} \times \mathcal{A}\right|=\frac{\dot{b}}{H} \mathcal{A} \ll 1 .
$$

Consequently, accumulation can be estimated locally from layer depth and age, through

$$
\dot{b} \approx \frac{h}{\mathcal{A}} .
$$

Combining Equations (24) and (25) shows that this approximation is valid only when $h / H \ll 1$ (e.g. in the upper few meters of a valley glacier, or in the upper 10-20 m (ice equivalent) of a thick ice sheet). The largest contributor to uncertainty can be imperfect knowledge of the density variation with depth. Pinglot and others (2001) used this approach for very shallow layers.

\section{Local-layer approximation (LLA)}

Where layers from several decameters depth or deeper are observed, the SLA (Equation (25)) cannot be used, because significant dynamic vertical strain has accumulated. Corrections based on a 1-D flow model are often applied at each site to account for vertical strain, i.e. the local vertical strain is used to approximate the strain encountered along an actual particle path. For example, if, following Morse and others (1999), we assume that the vertical velocity $w(z)$ is approximately linear with depth, such that the characteristic vertical strain rate $\dot{\epsilon}_{\text {char }}$ applies at all depths, then

$$
w(z) \approx-\dot{b} \frac{z}{H},
$$

which leads to the improved approximation

$\dot{b} \approx-\ln \left(1-\frac{h}{H}\right) \frac{H}{\mathcal{A}} \approx \frac{h}{\mathcal{A}}\left[1+\frac{1}{2}\left(\frac{h}{H}\right)+O\left(\left(\frac{h}{H}\right)^{2}\right)\right]$

When a local 1-D or sandwich model (Reeh, 1989) is used to make a strain correction, we call that process the LLA. For example, the LLA was used by Morse and others (1999), Fahnestock and others (2001), Kanagaratnam and others (2001) and Leysinger Vieli and others (2004).

\section{Deep layers}

We consider a layer to be 'deep' when the LLA is no longer applicable. For near-surface layers, the LLA may be as effective as the full inverse procedure that we have presented here, and less computationally intensive. For deeper layers, however, two effects warrant the use of a higher-dimensional inverse model.

First, in steady state, the downward ice velocity depends strongly on the local accumulation rate. Therefore, the depth of the layer at a particular point is a function of the average accumulation rate along the particle path, from its point of origin on the surface to the point where it intersects the measured layer (Fig. 5c). As the depth to an internal layer increases, a particle path is more likely to traverse regions of higher or lower accumulation rate, where the downward velocity pushes the path to a deeper or shallower trajectory, when compared with a path through a region of uniform accumulation rate. If the accumulation rate varies slowly with 
position, ice can follow a very long particle path, while still reaching a depth consistent with the LLA, based on the local accumulation rate at the end point. On the other hand, if the accumulation-rate gradient is large (as at Taylor Mouth), even moderate ice-flow velocities will cause internal-layer depths to differ significantly from the depth predicted with the LLA.

Second, as the depth of an internal layer increases to a larger fraction of the total ice thickness, the ice above that layer can be subjected to a greater total strain as a result of ice flow. Spatial variations of total vertical strain alter the depth of a layer, independent of the local accumulation rate, as demonstrated by Hindmarsh and others (2002). Variations in strain are commonly associated with spatial gradients in ice thickness. Large thickness gradients along a particle path can have a strong influence on layer depth at the end point of that path.

To decide whether horizontal gradients are important, we compare the total length $L_{\text {path }}$ of a particle path, to characteristic lengths, $L_{\dot{b}}$ and $L_{H}$, associated with accumulationrate and ice-thickness gradients, respectively. We express the particle-path length as

$$
L_{\text {path }}=\bar{U} \times \mathcal{A},
$$

where $\bar{U}$ is the temporally averaged horizontal velocity of a particle as it follows a particle path, and $\mathcal{A}$ is the age of the layer. A characteristic length $L_{\dot{b}}$, associated with the accumulation-rate gradient, is the length over which $\dot{b}$ changes by an appreciable fraction:

$$
\frac{1}{L_{\mathrm{b}}}=\left|\frac{1}{\dot{b}} \frac{\mathrm{d} \dot{b}}{\mathrm{~d} x}\right| .
$$

Similarly, a characteristic length $L_{\mathrm{H}}$ associated with the icethickness gradient is the length over which ice thickness changes by an appreciable fraction:

$$
\frac{1}{L_{H}}=\left|\frac{1}{H} \frac{\mathrm{d} H}{\mathrm{~d} x}\right| .
$$

When several processes act simultaneously or sequentially to modify a quantity of interest such as layer depth, we also want to characterize their combined effects. One way to do this is to combine characteristic numbers that represent each process individually, in ways that reflect the nature of those interactions. If the characteristic numbers describe difficulty in achieving an effect, or resistance to a process, they can be combined as serial and parallel resistors in a network, the total resistance of which is the characteristic value that we seek. This approach is used to study rates of aerosol deposition to environmental surfaces such as snow (e.g. Davidson, 1989). Waddington and others (2002) used this approach to explore the stability of stable-isotope ratios in polar snow. For our current question, a large characteristic length suggests that the depth to the internal layer varies slowly, so these characteristic lengths can be viewed as resistances to layer-depth variations. Because accumulation gradients and ice-thickness gradients act simultaneously, we can combine resistances $L_{\dot{b}}$ and $L_{H}$ in parallel to create a characteristic length scale $L_{\delta h}$, which represents total resistance to layerdepth variations from the combined effects of accumulation gradients and ice-thickness gradients:

$$
\frac{1}{L_{\delta \mathrm{h}}}=\frac{1}{L_{\mathrm{b}}}+\frac{1}{L_{\mathrm{H}}} \text {. }
$$

We now compare $L_{\text {path }}$ to $L_{\delta \mathrm{h}}$, to generate a non-dimensional number $D$ :

$$
D=\frac{L_{\text {path }}}{L_{\delta \mathrm{h}}} .
$$

If $D \ll 1$, then spatial gradients in $\dot{b}$ and $H$ do not significantly impact layer depth, and the LLA can be used. However, if $D \geq 1$, then layer depth at a point is not necessarily directly related to accumulation rate at that point, and a more sophisticated approach should be used. At Taylor Mouth, both $L_{\dot{b}}$ and $L_{\mathrm{H}}$ are important, and $D \sim 1$ for the layer that we used. This conclusion is confirmed by the differences between the initial and final accumulation rate and modelled layer (Fig. 6). Clearly the initial guess, which was the LLA, was inadequate, and a deep-layer treatment is required.

\subsection{Application to depth-age scales}

Whenever the LLA is inadequate to infer accumulation rate from a layer, use of a 1-D modelling approach to produce a depth-age scale for a core to similar depth is also questionable. We illustrate this point by comparing two depth-age scales for the Taylor Mouth core. The first is produced by our flowband model, while the second is produced by the following 1-D flow model.

\section{A 1-D flow model}

The depth-age relationship $\mathcal{A}(z)$ for a firn core can be estimated from the measured density profile $\rho(z)$ in the core if we make simplifying assumptions:

1. Firn compaction is a steady-state process that follows Sorge's law (Paterson, 1994, p. 14):

$$
\frac{\partial \rho}{\partial t}=0 \quad \text { and } \quad \frac{\partial w}{\partial t}=0 .
$$

2. The downward velocity $w(S)$ at the surface is equal to the net accumulation rate of snow at the site.

3. The accumulation rate is constant and uniform, i.e. independent of $t, x$ and $y$.

Then conservation of mass, $\nabla \cdot(\rho \mathbf{v})=0$, yields

$$
\frac{\mathrm{d} w(z)}{\mathrm{d} z}=-\frac{w(z)}{\rho(z)} \frac{\mathrm{d} \rho(z)}{\mathrm{d} z}-\dot{\varepsilon}_{\mathrm{H}},
$$

where $\dot{\varepsilon}_{\mathrm{H}}=(\partial u / \partial x)+(\partial v / \partial y)$ is the horizontal strain rate, which we assume is independent of depth over the length of the core, because horizontal velocity generally changes very little in the upper $20 \%$ of the ice column. Equation (34) can be integrated numerically from the surface, where $w(S)$ is known, to yield the vertical-velocity profile $w(z)$.

A second vertical integration,

$$
\mathcal{A}(z)=\int_{S}^{z} \frac{\mathrm{d} \eta}{w(\eta)},
$$

produces an estimate of the depth-age scale $\mathcal{A}(z)$ for the core. This analysis assumes that the area around the Taylor Mouth core site is horizontal. An identical analysis can be carried out for a sloping surface and a surface-normal core by rotating the coordinate system accordingly. Because the surface slope at Taylor Mouth is small ( 0.02), accounting for non-orthogonality of the surface and the core hole changed our calculated vertical velocity by $<0.1 \%$.

From repeated position measurements of the network of poles shown in Figure 3, the horizontal strain rate, $\dot{\varepsilon}_{\mathrm{H}}$, at 
the core site was determined to be $1.2 \times 10^{-4} \mathrm{a}^{-1}$. The depth-age scale derived from the 1-D compaction model with the measured accumulation rate at the core site $(0.023 \pm$ $0.010 \mathrm{~m} \mathrm{a}^{-1}$ ) is shown by the bold dashed black curve and the broad gray zone in Figure 9. This 1-D model can provide ages only down to the depth $(60 \mathrm{~m})$ at which the density of the core reaches the density of ice; the predicted age at $60 \mathrm{~m}$ is $2700 \pm 900$ years. When we repeated the calculation with $\dot{\varepsilon}_{\mathrm{H}}$ varied by $\pm 10 \%$, the calculated age varied by \pm 75 years at $60 \mathrm{~m}$ depth. Clearly, uncertainty in the accumulation measurement is the major contributor to uncertainty in depth-age, when using this 1-D model.

\section{Comparison with flowband model}

These results would form our best estimate of the depth-age scale, if we had no additional information from other locations along the flowline. However, the bold solid black curve and the associated hatched zone show the range of depthage scales from the 1-D compaction model when we used this 1-D model together with our new estimate of $0.036 \pm$ $0.009 \mathrm{~m} \mathrm{a}^{-1}$ (from Fig. 5a) for the 1662 year average accumulation rate at the core site. This calculation produced an age of $1610(+620,-350)$ years at $60 \mathrm{~m}$ depth

The 1-D model in Equation (35) is attractive in its simplicity. However, it cannot recognize the incompatibility of the measured (short-term) accumulation rate at the core site with respect to our (long-term) problem formulation (see section 6.3). As a result, it produced an error in age of 1090 years, or $68 \%$, at $60 \mathrm{~m}$ depth, when using the measured accumulation rate, rather than the inferred long-term accumulation rate at the site. In addition, the 1-D model took into account environmental conditions only at the core site. This model may yield reasonable estimates for short cores from environments with low spatial gradients of accumulation rate. Longer cores contain ice that originated some distance upstream, and this ice may have experienced different climate and flow conditions. The same criterion $D \ll 1$ (see Equation (32)) with which we assessed the validity of the LLA can also be used to assess the validity of a 1-D flow model applied to ice-core dating.

We also calculated a depth-age relationship (solid white curve in Fig. 9) for the core using the inferred accumulationrate profile (Fig. 5a) in our forward Taylor Mouth flowband model, and finding travel times of particles on paths that terminated at the core. As expected, both 1-D and 2-D models predict the same slope of the depth-age scale $\mathcal{A}(z)$ near the surface when both models use accumulation rates from Figure 5a. However, the two estimates diverge with depth, since $\mathcal{A}(z)$ from the flowband model incorporates upstream variations in accumulation rate and vertical strain. Since the accumulation rate increases upstream from the core site, the flowband model puts thicker layers in the upper $60 \mathrm{~m}$ and therefore puts younger ice at $60 \mathrm{~m}$ depth, where the two depth-age scales differ by 300 years, or approximately $18 \%$.

Because the flowband model can exploit all data contained in the layer geometry to constrain accumulation rate, its error limits on depth-age are smaller than those for the 1-D model. This analysis confirms our expectation, based on the non-dimensional analysis in section 6.5, that the depthage scale from the flowband model must be used for the Taylor Mouth ice core. More generally, the analysis confirms our expectation that non-dimensional analysis can determine whether a 1-D approach to calculation of a depth-age scale is valid or not for ice at any desired depth at any ice-core site.

\section{CONCLUSIONS}

Internal layers in polar ice sheets contain the only remaining detailed record of past accumulation rates; however, recovering that information requires integrated application of a thermomechanical ice-flow algorithm and formal inverse methods. The depths of near-surface layers in an ice sheet can provide a direct measurement of accumulation rate using the SLA, because they require neither complicated strain corrections nor knowledge of the total ice thickness. However, shallow layers can provide average accumulation rate only over short timescales in the recent past. For deeper, older layers for which vertical strain is appreciable, a local strain correction can be applied to derive an accumulationrate profile using the LLA. When internal-layer depths have been affected significantly by spatially variable ice strain and accumulation rate along particle trajectories, the LLA can be inadequate. The non-dimensional number, $D$, in Equation (32) determines when the LLA can be used, and when the full flowband particle-path treatment for deep layers is required. The same non-dimensional analysis can determine when a depth-age scale for an ice core can be derived with a 1-D analysis, and when spatial gradients of accumulation rate and ice thickness necessitate a full deep-layer treatment, with a 2-D or 3-D thermomechanical ice-flow algorithm.

When a deep-layer treatment is required, recovering the spatial pattern of accumulation rate should be posed as an inverse problem. We describe a general and versatile framework that incorporates a thermomechanical ice-flow algorithm to solve the forward problem, and uses radar data from a deep layer, together with other available data, including surface velocity, or accumulation-rate measurements in order to find a smooth accumulation-rate profile. Our procedure achieves an objective trade-off between solution smoothness and data fit, to avoid over-fitting the data.

To solve an inverse problem, we must solve the associated forward problem hundreds to thousands of times. To reduce computational time, the procedure used to solve the inverse problem should have the flexibility to make simplifications as necessary, while still capturing the essential physics of flow at each site. By describing ice velocities and particle paths in kinematic terms (see section 2.4), our approach offers the flexibility to choose the simplest thermomechanical iceflow model for each problem. Simplifications can include force-balance approximations such as the SIA; decoupling of the thermal and mechanical forward calculations; isolation of model parameters that are not strongly coupled to the other model parameters, or that are independently well known; and isolation of datasets that can be matched readily using the partial model-parameter solution for other model parameters.

Although it spans less than the upper $20 \%$ of the ice depth, the depth-age scale for the Taylor Mouth ice core is significantly affected by regions of relatively high accumulation rate upstream from the core site. That region of high accumulation correlates with a region of concave surface topography, in which both upslope and downslope wind speeds might be expected to decrease, dropping suspended and saltating snow.

In future work, the next step is to simultaneously infer both ice-sheet surface evolution and changing accumulation-rate patterns over time, by assimilating a series of both deep and shallow layers, using an objective trade-off criterion (Equation (11)). There is much still to be learned about our ability 
to resolve model parameters, and to assess the utility of the various data, as described in section 6.4 , for the long-term transient case.

\section{ACKNOWLEDGEMENTS}

We thank Antarctic Support Associates for field logistical support; J. Dibb, for gross- $\beta$ accumulation-rate measurements; M. Duval, for density measurements in snow pits; and P.M. Grootes and E.J. Steig, for density measurements on the Taylor Mouth core. H.C. Steen-Larsen contributed valuable insights on inverse problems. Reviewers F. Parrenin and D. MacAyeal, and Scientific Editor J. Meyssonnier provided many helpful comments and suggestions. This work was supported by US National Science Foundation grants OPP-8915924, OPP-9421644 and OPP-0440666.

\section{REFERENCES}

Appenzeller, C., T.F. Stocker and M. Anklin. 1998. North Atlantic Oscillation dynamics recorded in Greenland ice cores. Science, 282(5388), 446-449.

Aster, R., B. Borchers and C. Thurbur. 2004. Parameter estimation and inverse problems. New York, etc., Academic Press.

Baldwin, D.J., J.L. Bamber, A.J. Payne and R.L. Layberry. 2003. Using internal layers from the Greenland ice sheet, identified from radio-echo sounding data, with numerical models. Ann. Glaciol., 37, 325-330.

Bromwich, D.H., A.J. Monaghan and Z. Guo. 2004. Modeling the ENSO modulation of Antarctic climate in the late 1990s with the Polar MM5. J. Climate, 17(1), 109-132.

Conway, H., B.L. Hall, G.H. Denton, A.M. Gades and E.D. Waddington. 1999. Past and future grounding-line retreat of the West Antarctic ice sheet. Science, 286(5438), 280-283.

Dansgaard, W. and S.J. Johnsen. 1969. A flow model and a timescale for the ice core from Camp Century, Greenland. J. Glaciol., 8(53), 215-223.

Davidson, C.I. 1989. Mechanisms of wet and dry deposition of atmospheric contaminants to snow surfaces. In Oeschger, H. and C.C. Langway, Jr, eds. The environmental record in glaciers and ice sheets. Chichester, etc., John Wiley and Sons, 287-318.

Fahnestock, M.A., W. Abdalati, S. Luo and S. Gogineni. 2001. Internal layer tracing and age-depth-accumulation relationships for the northern Greenland ice sheet. J. Geophys. Res., 106(D24), 33,789-33,797.

Firestone, J., E. Waddington and J. Cunningham. 1990. The potential for basal melting under Summit, Greenland. J. Glaciol., 36(123), 163-168.

Frezzotti, M. and 13 others. 2005. Spatial and temporal variability of snow accumulation in East Antarctica from traverse data. J. Glaciol., 51(172), 113-124.

Frezzotti, M., S. Urbini, M. Proposito, C. Scarchilli and S. Gandolfi. 2007. Spatial and temporal variability of surface mass balance near Talos Dome, East Antarctica. J. Geophys. Res., 112(F2), F02032. (10.1029/2006JF000638.)

Gades, A.M. 1998. Spatial and temporal variations of basal conditions beneath glaciers and ice sheets inferred from radio echo soundings. (PhD thesis, University of Washington.)

Glen, J.W. 1958. The flow law of ice: a discussion of the assumptions made in glacier theory, their experimental foundation and consequences. IASH Publ. 47 (Symposium at Chamonix $1958-$ Physics of the Movement of the Ice), 171-183.

Grootes, P.M., E.J. Steig and M. Stuiver. 1994. Taylor ice dome study 1993-1994: an ice core to bedrock. Antarct. J. US, 29(5), 79-81.

Grootes, P.M., E.J. Steig, M. Stuiver, E.D. Waddington, D.L. Morse and M.J. Nadeau. 2001. The Taylor Dome Antarctic $\delta^{18} \mathrm{O}$ record and globally synchronous changes in climate. Quat. Res., 56(3), 289-298.

Hindmarsh, R.C., M.J. Siegert, H. Gudmundsson and D.L. Morse. 2002. Optimal fitting of radar-detected isochronic layers to models of tracer transport in ice sheets: extending cores sideways. [Abstract B21A-07.] Eos, 83(19).

Hvidberg, C.S. 1996. Steady-state thermomechanical modelling of ice flow near the centre of large ice sheets with the finite-element technique. Ann. Glaciol., 23, 116-123.

Indermuhle, A., E. Monnin, B. Stauffer, T. F. Stocker and M. Wahlen. 2000. Atmospheric $\mathrm{CO}_{2}$ concentration from 60 to $20 \mathrm{kyr}$ BP from the Taylor Dome ice core, Antarctica. Geophys. Res. Lett., 27(5), 735-738.

Kanagaratnam, P., S.P. Gogineni, N. Gundestrup and L. Larsen. 2001. High-resolution radar mapping of internal layers at the North Greenland Ice Core Project. J. Geophys. Res., 106(D24), 33,799-33,811.

King, J.C., P.S. Anderson, D.G. Vaughan, G.W. Mann, S.D. Mobbs and S.B. Vosper. 2004. Wind-borne redistribution of snow across an Antarctic ice rise. J. Geophys. Res., 109(D11), D11104. (10.1029/2003JD004361.)

Leonard, K., R.E. Bell, M. Studinger and B. Tremblay. 2004. Anomalous accumulation rates in the Vostok ice-core resulting from ice flow over Lake Vostok. Geophys. Res. Lett., 31(24) L24401. (10.1029/2004GL021102.)

Leysinger Vieli, G.J.M., M.J. Siegert and A.J. Payne. 2004. Reconstructing ice sheet accumulation rates at Ridge B, East Antarctica. Ann. Glaciol., 39, 326-330.

Martín, C., R.C.A. Hindmarsh and F.J. Navarro. 2006. Dating ice flow change near the flow divide at Roosevelt Island, Antarctica, by using a thermomechanical model to predict radar stratigraphy. J. Geophys. Res., 111(F1), F01011. (10.1029/2005JF000326.)

McConnell, J.R. and 7 others. 2000. Changes in Greenland ice sheet elevation attributed primarily to snow accumulation variability. Nature, 406(6798), 877-879.

Menke, W. 1989. Geophysical data analysis: discrete inverse theory. Revised edition. New York, etc., Academic Press.

Monnin, E. and 11 others. 2004. Evidence for substantial accumulation rate variability in Antarctica during the Holocene, through synchronization of $\mathrm{CO}_{2}$ in the Taylor Dome, Dome C and DML ice cores. Earth Planet. Sci. Lett., 224(1-2), 45-54.

Morse, D.L. 1997. Glacier geophysics at Taylor Dome, Antarctica. (PhD thesis, University of Washington.)

Morse, D.L., E.D. Waddington and E.J. Steig. 1998. Ice age storm trajectories inferred from radar stratigraphy at Taylor Dome, Antarctica. Geophys. Res. Lett., 25(17), 3383-3386.

Morse, D.L. and 7 others. 1999. Accumulation rate measurements at Taylor Dome, East Antarctica: techniques and strategies for mass balance measurements in polar environments. Geogr. Ann., 81A(4), 683-694.

Morse, D.L., E.D. Waddington and L.A. Rasmussen. 2007. Ice deformation in the vicinity of the ice core site at Taylor Dome, Antarctica, and a derived accumulation-rate history. J. Glaciol., 53(182), 449-460.

Morse, P.M. and H. Feshbach. 1953. Methods of theoretical physics, part 1. New York, McGraw-Hill.

Nereson, N.A. and C.F. Raymond. 2001. The elevation history of ice streams and the spatial accumulation pattern along the Siple Coast of West Antarctica inferred from ground-based radar data from three inter-ice-stream ridges. J. Glaciol., 47(157), 303-313.

Nereson, N.A. and E.D. Waddington. 2002. Isochrones and isotherms beneath migrating ice divides. J. Glaciol., 48(160), 95-108.

Nereson, N.A., C.F. Raymond, E.D. Waddington and R.W. Jacobel. 1998. Migration of the Siple Dome ice divide, West Antarctica. J. Glaciol., 44(148), 643-652. 
Nereson, N.A., C.F. Raymond, R.W. Jacobel and E.D. Waddington. 2000. The accumulation pattern across Siple Dome, West Antarctica, inferred from radar-detected internal layers. J. Glaciol., 46(152), 75-87.

Neumann, T.A., E.D. Waddington, E.J. Steig and P.M. Grootes. 2005. Non-climate influences on stable isotopes at Taylor Mouth, Antarctica. J. Glaciol., 51(173), 248-252.

Noone, D. and I. Simmonds. 2002. Annular variations in moisture transport mechanisms and the abundance of $\delta^{18} \mathrm{O}$ in Antarctic snow. J. Geophys. Res., 107(D24), 4742-4754.

Pälli, A. and 6 others. 2002. Spatial and temporal variability of snow accumulation using ground-penetrating radar and ice cores on a Svalbard glacier. J. Glaciol., 48(162), 417-424.

Paren, J.G. and G.D. Robin. 1975. Internal reflections in polar ice sheets. J. Glaciol., 14(71), 251-259.

Parker, R.L. 1994. Geophysical inverse theory. Princeton, NJ, Princeton University Press.

Parrenin, F., R.C.A. Hindmarsh and F. Rémy. 2006. Analytical solutions for the effect of topography, accumulation rate and lateral flow divergence on isochrone layer geometry. J. Glaciol., 52(177), 191-202.

Paterson, W.S.B. 1994. The physics of glaciers. Third edition. Oxford, etc., Elsevier.

Picciotto, E. and S. Wilgain. 1963. Fission products in Antarctic snow, a reference level for measuring accumulation. J. Geophys. Res., 68(21), 5965-5972.

Pinglot, J.F., J.O. Hagen, K. Melvold, T. Eiken and C. Vincent. 2001. A mean net accumulation pattern derived from radioactive layers and radar soundings on Austfonna, Nordaustlandet, Svalbard. J. Glaciol., 47(159), 555-566.

Press, W.H., B.P. Flannery, S.A. Teukolsky and W.T. Vetterling. 1986. Numerical recipes: the art of scientific computing. Cambridge, etc., Cambridge University Press.

Raymond, C.F. 1983. Deformation in the vicinity of ice divides. J. Glaciol., 29(103), 357-373.

Reeh, N. 1988. A flow-line model for calculating the surface profile and the velocity, strain-rate, and stress fields in an ice sheet. J. Glaciol., 34(116), 46-54.

Reeh, N. 1989. The age-depth profile in the upper part of a steady-state ice sheet. J. Glaciol., 35(121), 406-417.

Siegert, M.J. and A.J. Payne. 2004. Past rates of accumulation in central West Antarctica. Geophys. Res. Lett., 31(12), L12403. (10.1029/2004GL020290.)

Spikes, V.B., G.S. Hamilton, S.A. Arcone, S. Kaspari and P. Mayewski. 2004. Variability in accumulation rates from GPR profiling on the West Antarctic plateau. Ann. Glaciol., 39, 238-244.

Steig, E.J. 1996. Beryllium-10 in the Taylor Dome ice core: applications to Antarctic glaciology and paleoclimatology. (PhD thesis, University of Washington.)

Steig, E.J., C.P. Hart, J.W.C. White, W.L. Cunningham, M.D. Davis and E.S. Saltzman. 1998. Changes in climate, ocean and ice-sheet conditions in the Ross embayment, Antarctica, at $6 \mathrm{ka}$. Ann. Glaciol., 27, 305-310.

Steig, E.J. and 7 others. 2000. Wisconsinan and Holocene climate history from an ice core at Taylor Dome, western Ross Embayment, Antarctica. Geogr. Ann., 82A(2-3), 213-235.

Truffer, M. 2004. The basal speed of valley glaciers: an inverse approach. J. Glaciol., 50(169), 236-242.

Van der Veen, C.J. 1993. Interpretation of short-time ice-sheet elevation changes inferred from satellite altimetry. Climatic Change, 23(4), 383-405.

Vaughan, D.G., H.F.J. Corr, C.S.M. Doake and E.D. Waddington. 1999. Distortion of isochronous layers in ice revealed by ground-penetrating radar. Nature, 398(6725), 323-326.

Waddington, E.D. 1982. Accurate modelling of glacier flow. (PhD thesis, University of British Columbia.)
Waddington, E.D., E.J. Steig and T.A. Neumann. 2002. Using characteristic times to assess whether stable isotopes can be reversibly deposited. Ann. Glaciol., 35, 118-124.

Weertman, B.R. 1993. Interpretation of ice sheet stratigraphy: a radio-echo sounding study of the Dyer Plateau, Antarctica. (PhD thesis, University of Washington.)

\section{APPENDIX A \\ LEAST-SQUARES SOLUTION PROCEDURE}

Using Equations (17) and (19), the performance index in Equation (12) can be written as

$$
I_{p}=\sum_{i=1}^{N_{\mathrm{p}}}\left(w_{i}^{(\mathrm{c})}\right)^{2} c_{i}^{2}+\sum_{i=1}^{N_{\mathrm{d}}}\left(w_{i}^{(\mathrm{r})}\right)^{2} r_{i}^{2}-\nu T^{2},
$$

where $N_{\mathrm{p}}$ is the number of model parameters, and $N_{\mathrm{d}}=$ $N_{h}+N_{S}+N_{b}+N_{u}$ is the total number of data, comprising the layer-depth profile, surface-elevation profile, and any available measurements of accumulation rate and surface velocity. The positive weights $\left(w_{i}^{(r)}\right)^{2}$ are all equal to the trade-off parameter $\nu$. The goal is to find a vector of parameters $p_{j}$ that minimize $I_{p}$ for a given value of $\nu$. The model residuals $c_{i}$ depend on the model parameters $p_{j}$, through Equations (15) and (16), and the data residuals $r_{i}$ depend on the model parameters $p_{j}$, through the model predictions $o_{i}^{(\mathrm{m})}$ of observable quantities in Equation (18). Starting from estimates $p_{j}^{(0)}$ of the parameters, we calculate trial values $c_{i}^{(0)}$ of the model residuals and $r_{i}^{(0)}$ of the data residuals. These values, when substituted into Equation (A1) along with an estimate $\nu^{(0)}$ of the trade-off parameter, are unlikely to produce a minimum in $I_{p}$. However, we can approximate the unknown values of the model residuals $c_{i}$ and data residuals $r_{i}$ that would minimize $I_{p}$ with $\nu=\nu^{(0)}$ by expanding $c_{i}$ and $r_{i}$ in the vicinity of their current estimates, $c_{i}^{(0)}$ and $r_{i}^{(0)}$ :

$$
\begin{gathered}
c_{i}=c_{i}^{(0)}+\sum_{k=1}^{N_{\mathrm{p}}} \frac{\partial c_{i}}{\partial p_{k}} \Delta p_{k} \\
r_{i}=r_{i}^{(0)}+\sum_{k=1}^{N_{\mathrm{p}}} \frac{\partial r_{i}}{\partial p_{k}} \Delta p_{k} .
\end{gathered}
$$

Substituting Equation (A2) into Equation (A1) produces

$$
\begin{gathered}
I_{p}=\left[\sum_{i=1}^{N_{\mathrm{p}}}\left(w_{i}^{(\mathrm{c})}\right)^{2}\left(c_{i}^{(0)}\right)^{2}+\sum_{i=1}^{N_{\mathrm{d}}}\left(w_{i}^{(\mathrm{r})}\right)^{2}\left(r_{i}^{(0)}\right)^{2}-\nu^{(0)} T^{2}\right] \\
+2\left[\sum_{i=1}^{N_{\mathrm{p}}}\left(w_{i}^{(\mathrm{c})}\right)^{2} c_{i}^{(0)} \sum_{k=1}^{N_{\mathrm{p}}} \frac{\partial c_{i}}{\partial p_{k}} \Delta p_{k}\right. \\
\left.+\sum_{i=1}^{N_{\mathrm{d}}}\left(w_{i}^{(\mathrm{r})}\right)^{2} r_{i}^{(0)} \sum_{k=1}^{N_{\mathrm{p}}} \frac{\partial r_{i}}{\partial p_{k}} \Delta p_{k}\right] \\
+\left[\sum_{i=1}^{N_{\mathrm{d}}}\left(w_{i}^{(\mathrm{C})}\right)^{2}\left(\sum_{k=1}^{N_{\mathrm{p}}} \frac{\partial c_{i}}{\partial p_{k}} \Delta p_{k}\right)^{2}\right. \\
\left.+\sum_{i=1}^{N_{\mathrm{d}}}\left(w_{i}^{(\mathrm{r})}\right)^{2}\left(\sum_{k=1}^{N_{\mathrm{p}}} \frac{\partial r_{i}}{\partial p_{k}} \Delta p_{k}\right)^{2}\right] .
\end{gathered}
$$


Because model residuals $c_{i}$ and data residuals $r_{i}$ appear in similar forms, we can simplify the notation by combining them into a single column vector $\mathbf{e}$

$$
\mathbf{e}=-\left[\begin{array}{l}
\mathbf{c} \\
\mathbf{r}
\end{array}\right] \text {. }
$$

We also introduce the $N_{p} \times N_{p}$ diagonal weight matrix $\mathbf{W}_{c}$, whose $i$ th diagonal element is $w_{i}^{(\mathrm{c})}$, and the $N_{\mathrm{d}} \times N_{\mathrm{d}}$ diagonal weight matrix $\mathbf{W}_{\mathrm{r}}$, whose $i$ th diagonal element is $w_{i}^{(\mathrm{r})}=\nu^{1 / 2}$. We can then form a $\left(N_{p}+N_{d}\right) \times\left(N_{p}+N_{d}\right)$ diagonal weight matrix

$$
\mathbf{W}=\left[\begin{array}{cc}
\mathbf{W}_{\mathrm{c}} & \mathbf{0} \\
\mathbf{0} & \mathbf{W}_{\mathrm{r}}
\end{array}\right]
$$

Finally, we form a $N_{p} \times N_{p}$ Jacobian matrix $\mathbf{J}_{c}$, whose element in row $i$ and column $j$ is the partial derivative $\partial c_{i} / \partial p_{j}$, and a $N_{\mathrm{d}} \times N_{\mathrm{p}}$ Jacobian matrix $\mathbf{J}_{\mathrm{r}}$, whose element in row $i$ and column $j$ is the partial derivative $\partial r_{i} / \partial p_{j}$. From these two matrices we form a global $\left(N_{p}+N_{d}\right) \times N_{p}$ Jacobian matrix, J:

$$
\mathbf{J}=\left[\begin{array}{l}
\mathbf{J}_{\mathrm{c}} \\
\mathbf{J}_{\mathrm{r}}
\end{array}\right] \text {. }
$$

The minimization Equation (13) becomes:

$$
\begin{aligned}
\frac{\partial I_{p}}{\partial p_{j}}=2 & {\left[\sum_{i=1}^{N_{\mathrm{p}}+N_{\mathrm{d}}}\left(W_{i i}^{2} e_{i}^{(0)} \frac{\partial e_{i}}{\partial p_{j}}\right)\right.} \\
& \left.+\sum_{i=1}^{N_{\mathrm{p}}+N_{\mathrm{d}}} \sum_{k=1}^{N_{\mathrm{p}}}\left(W_{i i}^{2} \frac{\partial e_{i}}{\partial p_{j}} \frac{\partial e_{i}}{\partial p_{k}} \Delta p_{k}\right)\right]=0 .
\end{aligned}
$$

We can now express the complete set of these $N_{p}$ so-called normal equations for the least-squares solution in the matrix form

$$
\mathbf{J}^{\mathrm{T}} \mathbf{W}^{\mathrm{T}} \mathbf{W} \mathbf{J} \Delta \mathbf{p}=\mathbf{J}^{\mathrm{T}} \mathbf{W}^{\mathrm{T}} \mathbf{W} \mathbf{e},
$$

the solution of which is

$$
\Delta \mathbf{p}=\left[\mathbf{J}^{\mathbf{T}} \mathbf{W}^{\mathbf{T}} \mathbf{W} \mathbf{J}\right]^{-1} \mathbf{J}^{\mathbf{T}} \mathbf{W}^{\mathbf{T}} \mathbf{W} \mathbf{e} .
$$

Defining a $\left(N_{p}+N_{d}\right) \times N_{p}$ matrix $\mathbf{A}=\mathbf{W}$ J, and a column vector $\mathbf{b}=\mathbf{W} \mathbf{e}$, of length $\left(N_{p}+N_{d}\right)$, we can express Equation (A8) as

$$
\mathbf{A}^{\mathrm{T}} \mathbf{A} \Delta \mathbf{p}=\mathbf{A}^{\mathrm{T}} \mathbf{b},
$$

which has the standard form of the normal equations of the least-squares problem of finding the vector $\Delta \mathbf{p}$ that minimizes the quadratic form $|\mathbf{A} \Delta \mathbf{p}-\mathbf{b}|^{2}$ (e.g. Press and others, 1986, p. 512).

However, the solution expressed in the form of Equation (A9) can be unstable or non-existent, if the matrix $\mathbf{A}^{\mathbf{T}} \mathbf{A}=$ $\mathbf{J}^{\mathbf{T}} \mathbf{W}^{\mathbf{T}} \mathbf{W} \mathbf{J}$ is near-singular or singular. To avoid this problem, we use the singular value decomposition (SVD; e.g. Press and others, 1986, p. 52) of matrix A, which in its reduced form is

$$
\mathbf{A}=\mathbf{U} \boldsymbol{\Lambda} \mathbf{V}^{\mathbf{T}}
$$

where $\mathbf{V}$ is a $N_{p} \times N_{p}$ matrix whose columns $\mathbf{v}_{(i)}(i=1, \ldots$, $\left.N_{p}\right)$, are orthonormal basis vectors that span the $N_{p}$-dimensional parameter space of all possible vectors $\Delta \mathbf{p}$. The vector $\mathbf{b}$ exists in a $\left(N_{p}+N_{d}\right)$-dimensional space of residuals. However, the matrix $\mathbf{A}$, by multiplying any vector $\Delta \mathbf{p}$, can produce only vectors $\mathbf{b}$ that lie within an $N_{p}$-dimensional sub-space known as the range of $\mathbf{A}$. The columns $\mathbf{u}_{(i),}(i=$ $\left.1, \ldots, N_{p}\right)$ of the non-square $\left(N_{p}+N_{d}\right) \times N_{p}$ matrix $\mathbf{U}$ are orthonormal basis vectors that span the range of $\mathbf{A}$ within that $\left(N_{p}+N_{d}\right)$-dimensional residuals space. Finally, $\boldsymbol{\Lambda}$ is a $N_{p} \times N_{p}$ diagonal matrix whose diagonal elements $\lambda_{i i}$ are called the singular values, which are also the square roots of the eigenvalues of $\mathbf{A}^{\mathbf{T}} \mathbf{A}$.

Because columns of $\mathbf{U}$ and $\mathbf{V}$ are orthonormal, $\mathbf{U}^{\mathbf{T}} \mathbf{U}$ and $\mathbf{V}^{\mathbf{T}} \mathbf{V}$ produce identity matrices, and because $\boldsymbol{\Lambda}$ is diagonal, $\boldsymbol{\Lambda}^{\mathbf{T}}=\boldsymbol{\Lambda}$, and its inverse $\boldsymbol{\Lambda}^{-1}$ is also a $N_{p} \times N_{p}$ diagonal matrix, with $1 / \lambda_{i i}$ as the $i$ th diagonal element. With these simplifications, Equation (A8) reduces to

$$
\left[\mathbf{U} \boldsymbol{\Lambda} \mathbf{V}^{\mathbf{T}}\right] \Delta \mathbf{p}=\mathbf{A} \Delta \mathbf{p}=\mathbf{b},
$$

and the solution Equation (A9) reduces to

$$
\Delta \mathbf{p}=\left[\mathbf{V} \boldsymbol{\Lambda}^{-1} \mathbf{U}^{\mathbf{T}}\right] \mathbf{b}=\left[\mathbf{V} \boldsymbol{\Lambda}^{-1} \mathbf{U}^{\mathbf{T}} \mathbf{W}\right] \mathbf{e} .
$$

The $N_{p} \times\left(N_{p}+N_{d}\right)$ matrix $\left[\mathbf{V} \Lambda^{-1} \mathbf{U}^{\mathbf{T}}\right]$ is known as the generalized inverse of the $\left(N_{p}+N_{d}\right) \times N_{p}$ matrix $\mathbf{A}=\mathbf{W}$ J.

SVD helps us to find a solution when $\mathbf{A}^{\mathbf{T}} \mathbf{A}$ is ill-conditioned. Equation (A12) defining the problem can also be written in terms of the basis vectors $\mathbf{u}_{(i)}$ and $\mathbf{v}_{(i)}$ as

$$
\mathbf{e}=\sum_{i=1}^{N_{\mathrm{p}}}\left(\lambda_{i i} \mathbf{v}_{(i)} \cdot \Delta \mathbf{p}\right) \mathbf{u}_{(i)}
$$

When the vector e (representing the known model residuals and data residuals) is written as a linear combination of the basis vectors $\mathbf{u}_{(i)}$ spanning the range of $\mathbf{A}$ in the residuals space, the coefficient of each $\mathbf{u}_{(i)}$ is given by the projection $\mathbf{v}_{(i)} \cdot \Delta \mathbf{p}$ of the parameter-correction vector onto the corresponding ith basis vector in the parameter space, weighted by the $i$ th singular value. If any parameter-change vector $\Delta \mathbf{p}$ lies entirely along a particular $\mathbf{v}_{(i)}$, then the residual vector that it produces lies entirely along $\mathbf{u}_{(i)}$ in the residuals space. If the weight $\lambda_{i i}$ is small, then the component of $\Delta \mathbf{p}$ aligned with the corresponding basis vector $\mathbf{v}_{(i)}$ has very little influence on the residual vector $\mathbf{e}$, in the sense that the matrix $\mathbf{A}$ maps it onto some very small vector $\mathbf{b}$. When the physical processes in the forward algorithm (represented by $\mathbf{A}$ ) are integrative or diffusive, the basis vectors $\mathbf{v}_{(i)}$ associated with small $\lambda_{i i}$ tend to be highly oscillatory, and the oscillations tend to cancel when $\mathbf{v}_{(i)}$ is mapped to the residuals space by $\mathbf{A}$.

The solution vector $\Delta \mathbf{p}$ in Equation (A13) can also be written as a linear combination of the basis vectors $\mathbf{v}_{(i)}$ in the parameter space (Press and others, 1986, p. 516),

$$
\Delta \mathbf{p}=\sum_{i=1}^{N_{\mathrm{p}}}\left(\frac{\mathbf{u}_{(i)} \cdot \mathbf{e}}{\lambda_{i i}}\right) \mathbf{v}_{(i)},
$$

and the coefficient of each basis vector $\mathbf{v}_{(i)}$ is the projection of the residuals vector $\mathbf{b}$ onto the corresponding basis vector $\mathbf{u}_{(i)}$ in the range of $\mathbf{A}$, weighted by the inverse of the corresponding singular value $\lambda_{i i}$. Near-zero or zero singular values $\lambda_{i i}$ produce large or infinite weights $1 / \lambda_{i i}$. Any component of the residual vector that lies along $\mathbf{u}_{(i)}$ will make a very large (or infinite) contribution to the solution $\Delta \mathbf{p}$, through its corresponding (and often highly oscillatory) unit vector $\mathbf{v}_{(i)}$ in the parameter space.

The standard remedy (e.g. Press and others, 1986, p. 56) is to set to zero each large diagonal element $1 / \lambda_{i i}$ in $\boldsymbol{\Lambda}^{-1}$ that may have been corrupted by round-off errors. This prevents the solution procedure from attempting to 'explain' the component of the residuals vector $\mathbf{e}$ that is aligned with the 
corresponding residuals basis vector $\mathbf{u}_{(i)}$ by adding a very large multiple of the possibly oscillatory corresponding basis vector $\mathbf{v}_{(i)}$ to the solution $\Delta \mathbf{p}$. Leaving a part of the residuals 'unexplained' is generally preferable to polluting the solution with a large and generally meaningless component. In practice, our regularization through the smoothness criterion (Equations (15) and (17)), tends to keep A well conditioned, and singular values are only occasionally too small.

\section{APPENDIX B}

\section{FLOW MODEL FOR TAYLOR MOUTH}

\section{Velocity and temperature calculations}

We assume that ice deformation follows a temperaturedependent Glen-type flow law (Glen, 1958). In the Taylor Mouth flowband, we use the SIA (e.g. Paterson, 1994, p. 262), in which the dominant deviatoric-stress component in Equation (1) is surface-parallel shear stress $\tau_{\mathrm{xz}}$, so that Equation (1) reduces to

$$
\frac{\partial u}{\partial z}=2 E A(\theta)\left(-\rho g \frac{\mathrm{d} S(x)}{\mathrm{d} x}\right)^{n}(S(x)-z)^{n},
$$

where $\rho$ is ice density, $g$ is gravitational acceleration, $n=3$ is the flow-law exponent, and $\theta$ is temperature. $A(\theta)$ is a temperature-dependent softness or fluidity parameter, as described in section 2.2. While longitudinal stresses may be comparable in some places along the flowband, particularly in the deep ice near the steep bedrock slope at $x=3.5 \mathrm{~km}$, their impact on particle paths in the upper $20 \%$ of the ice depth should be small.

In our flowband model for Taylor Mouth, the rheological behavior of ice is fully determined by two model parameters, the enhancement factor $E$ and the geothermal flux $q_{g e o}$. We decouple $E, H_{0}$ (ice thickness at the core site) and $q_{\text {geo }}$ from the other model parameters, and treat $E$ as a spatially uniform adjustable parameter (Fig. 7). We treat $q_{\text {geo }}$ as a constant, using the value determined nearby at Taylor Dome (Table 1).

At each $x$, we then calculate the velocity $u(x, \hat{z})$ as a function of non-dimensional height $\hat{z}$ using Equation (5) to replace $z$ by $\hat{z}$, and integrating Equation (B1) from the bed at $\hat{z}=0$, to elevation $\hat{z}$ :

$$
\begin{aligned}
u(x, \hat{z})= & \bar{u}(x) \phi(x, 0)+2\left(-\rho g \frac{\mathrm{d} S(x)}{\mathrm{d} x}\right)^{n} H(x)^{n+1} \\
& \cdot \int_{0}^{\hat{z}} E(x, \hat{\zeta}) A(\theta(x, \hat{\zeta}))(1-\hat{\zeta})^{n} \mathrm{~d} \hat{\zeta}
\end{aligned}
$$

Equation (B2) can be written in the form of Equation (4), with

$$
\begin{aligned}
\bar{u}(x)= & 2\left(-\rho g \frac{\mathrm{d} S(x)}{\mathrm{d} x}\right)^{n} \frac{H(x)^{n+1}}{1-\phi(x, 0)} \\
& \cdot \int_{0}^{1} \int_{0}^{\hat{z}} E(x, \hat{\zeta}) A(\theta(x, \hat{\zeta}))(1-\hat{\zeta})^{n} \mathrm{~d} \hat{\zeta} \mathrm{d} \hat{z},
\end{aligned}
$$

and the horizontal-velocity shape function given by:

$$
\phi(x, \hat{z})=\frac{u(x, \hat{z})}{\bar{u}(x)} .
$$

For the special case of an isothermal vertical column of ice with temperature $\theta_{0}$, no sliding $(\phi(x, 0)=0)$, no melting $(\dot{m}(x)=0)$, and uniform $E$ :

$$
\begin{aligned}
\bar{u}(x) & =\frac{1}{n+2} 2 E A\left(\theta_{0}\right)\left(-\rho g \frac{\mathrm{d} S}{\mathrm{~d} x}\right)^{n} H(x)^{n+1} \\
\phi(x, \hat{z}) & =\frac{n+2}{n+1}\left[1-(1-\hat{z})^{n+1}\right] .
\end{aligned}
$$

Finally, to evaluate $\phi(x, \hat{z})$ with vertical temperature gradients, we calculate the temperature profile $\theta(x, z)$ at each node $x_{i}^{(\mathrm{n})}$ with the 1-D steady-state temperature model of Firestone and others (1990), using estimated mean annual surface temperatures (interpolated from measured $10 \mathrm{~m}$ firn temperatures on the flowline), and estimated regional geothermal flux measured in the Taylor Dome borehole $20 \mathrm{~km}$ away (personal communication from G.D. Clow, 1999; see Table 1) as boundary conditions. Measured accumulation rates are only a few cm (ice equivalent) annually, so vertical velocities are also small. With characteristic ice thickness of $700 \mathrm{~m}$, and thermal diffusivity $\kappa \approx 10^{-6} \mathrm{~m}^{2} \mathrm{~s}^{-1}$, the Péclet number (e.g. Nereson and Waddington, 2002) is $\mathrm{Pe}=H \dot{b} / \kappa \approx 0.5$ at Taylor Mouth, implying that neither horizontal advection nor errors in calculated vertical advection have an appreciable impact on the near-linear vertical temperature profiles. At sites with larger Péclet numbers, a more extensive treatment of temperature may be necessary. The bedrock is cold, so we can assume no melting and no sliding.

\section{Surface topography}

When the flowband width $W(x)$ and the bedrock height $B(x)$ are known and the temperature field has been estimated as above, we can calculate a modelled ice-sheet surface elevation $S(x)$ in two additional steps. First, we equate the depth-averaged ice velocity (Equation (B3)) using a temperature-dependent softness parameter, to the depthaveraged velocity (Equation (B5)) calculated for an isothermal column with temperature $\theta_{0}(x)$, and we solve for the effective isothermal softness parameter $A\left(\theta_{\mathrm{O}}(x)\right)$ at each position $x$ :

$$
A\left(\theta_{\mathrm{O}}(x)\right)=(n+2) \int_{0}^{1} \int_{0}^{\hat{z}} A(\theta(x, \hat{\zeta}))(1-\hat{\zeta})^{n} \mathrm{~d} \hat{\zeta} \mathrm{d} \hat{z} .
$$

Second, using Equation (3) to replace $\bar{u}(x)$ by $q(x) /$ $[H(x) W(x)]$, and recalling that $H(x)=S(x)-B(x)$, we rewrite Equation (B5) as an ordinary differential equation for the icesurface elevation, $S(x)$, along the flowband:

$$
\frac{\mathrm{d} S(x)}{\mathrm{d} x}=-\left[\frac{(n+2) q(x)}{2 E A\left(\theta_{\mathrm{O}}(x)\right)(\rho g)^{n} W(x)(S(x)-B(x))^{n+2}}\right]^{1 / n} .
$$

This equation can be solved numerically using Runge-Kutta methods (e.g. Press and others, 1986, p. 550) for the icesurface elevation $S(x)$ when the ice flux $q(x)$ along the flowband is known from Equation (2). A known ice thickness at a single point along the flowband provides the necessary boundary condition. For example, the measured elevation at the core site allows us to integrate Equation (B8) both upstream and downstream. 